\title{
General structure of fermion two-point function and its spectral representation in a hot magnetized medium
}

\author{
Aritra Das ${ }^{*}$ \\ HENPP Division, Saha Institute of Nuclear Physics, HBNI, 1/AF Bidhan Nagar, Kolkata 700064, India \\ Aritra Bandyopadhyay ${ }^{\dagger}$ \\ Theory Division, Saha Institute of Nuclear Physics, HBNI, 1/AF Bidhan Nagar, Kolkata 700064, India \\ Departamento de Fisica, Universidade Federal de Santa Maria, Santa Maria, RS 97105-900, Brazil \\ Pradip K. Roy \\ HENPP Division, Saha Institute of Nuclear Physics, HBNI, 1/AF Bidhan Nagar, Kolkata 700064, India \\ Munshi G. Mustafa ${ }^{\S}$ \\ Theory Division, Saha Institute of Nuclear Physics, HBNI, 1/AF Bidhan Nagar, Kolkata 700064, India
}

(Received 10 November 2017; published 22 February 2018)

\begin{abstract}
We have systematically constructed the general structure of the fermion self-energy and the effective quark propagator in the presence of a nontrivial background such as a hot magnetized medium. This is applicable to both QED and QCD. The hard thermal loop approximation has been used for the heat bath. We have also examined transformation properties of the effective fermion propagator under some of the discrete symmetries of the system. Using the effective fermion propagator we have analyzed the fermion dispersion spectra in a hot magnetized medium along with the spinor for each fermion mode obtained by solving the modified Dirac equation. The fermion spectra is found to reflect the discrete symmetries of the two-point functions. We note that for a chirally symmetric theory the degenerate left- and right-handed chiral modes in vacuum or in a heat bath get separated and become asymmetric in the presence of a magnetic field without disturbing the chiral invariance. The obtained general structure of the two-point functions is verified by computing the three-point function, which agrees with the existing results in oneloop order. Finally, we have computed explicitly the spectral representation of the two-point functions which would be very important to study the spectral properties of the hot magnetized medium corresponding to QED and QCD with background magnetic field.
\end{abstract}

DOI: $10.1103 /$ PhysRevD.97.034024

\section{INTRODUCTION}

In noncentral heavy ion collision (HIC) experiments in LHC at CERN and in RHIC at BNL, it is believed that a very strong magnetic field is created in the direction perpendicular to the reaction plane due to the spectator particles that are not participating in the collisions. The experiments conducted by PHENIX Collaboration [1] showed direct-photon anisotropy which has posed a serious

\footnotetext{
*aritra.das@saha.ac.in

†ritra.bandyopadhyay@saha.ac.in

*pradipk.roy@saha.ac.in

\$munshigolam.mustafa@saha.ac.in
}

Published by the American Physical Society under the terms of the Creative Commons Attribution 4.0 International license. Further distribution of this work must maintain attribution to the author(s) and the published article's title, journal citation, and DOI. Funded by SCOAP ${ }^{3}$. challenge to the present theoretical models. It is conjectured that this excess elliptic flow may be due to the excess photons produced by the decay $\rho \rightarrow \pi(\eta) \gamma$ and the branching ratio of which increases in the presence of the magnetic field near the critical value where the condensate of $\rho$ is found. The estimated strength of this magnetic field depends on collision energy and impact parameter between the colliding nuclei and is about several times the pion mass squared, i.e., $e B \sim 15 m_{\pi}^{2}$ at LHC in CERN [2]. Also, a class of neutron star called magnetar exhibits [3-5] a magnetic field of $10^{18}-10^{20}$ Gauss at the inner core and $10^{12}-10^{13}$ Gauss at the surface. These observations motivate to study the properties of hot magnetized medium using both phenomenology and quantum field theory.

The presence of a strong magnetic field in HIC influences the QCD phase transitions [6] and particle productions, especially the production of the soft photon [7] and dileptons [8-13], which act as a probe of the 
medium. Apart from these, there is a large class of other phenomena that take place in the presence of a background magnetic field such as chiral magnetic effects due to axial anomalies [14-16], magnetic catalysis [17,18], inverse magnetic catalysis $[19,20]$, and superconductivity of the vacuum [21]. It further influences the thermal chiral and deconfining phase transition [22], change of topological charge [23], anomalous transports [24], refractive indices $[25,26]$ and screening mass [27], and decay constant [28] of neutral mesons, etc. In addition, efforts were also made to study the bulk properties for a Fermi gas [29], low lying hadrons [30] and strongly coupled systems [31], collective excitations in magnetized QED medium [32] using Ritus method and QCD medium [33] using Furry's picture, neutrino properties [34,35] and field theory of the Faraday effects [36,37].

The magnetic field created in HIC lasts for very short time $(\sim$ a few $\mathrm{fm})$. The strength of the field decays rapidly with time after $\tau \sim 1-2 \mathrm{fm} / \mathrm{c}$. However, the medium effects such as electric conductivity can delay the decay and by the time deconfined quarks and gluons equilibrate with QGP medium, the magnetic field strength gets sufficiently weak. At that time the relevant energy scales of the system can be put in this way: $q_{f} B<m_{\pi}^{2} \ll T^{2}$. In this low-field limit, the properties of the deconfined medium are also affected. So, it becomes important to treat the weak-field limit separately. Fermion propagator in the presence of a uniform background magnetic field has been derived first by Schwinger [38]. Using this, one-loop fermion self-energy and the vacuum polarization was calculated in double parameter integral in $[39,40]$, respectively. The weak-field expansion of this propagator was calculated order by order in powers of $q_{f} B$ in [41]. Recently, the pion self-energy and its dispersion property have been studied at zero temperature [42] in weak-field approximation and using full propagator at finite temperature [43]. Also a detailed study of the spectral properties of $\rho$ mesons has been performed in the presence of a magnetic field both at zero $[44,45]$ and at nonzero temperature [46].

For a hot and dense medium (e.g., QED and QCD plasma), it is well known that a bare perturbation theory breaks down due to infrared divergences. A reorganization of the perturbation theory has been done by performing the expansion around a system of massive quasiparticles [47], where mass is generated through thermal fluctuations. This requires a resummation of a certain class of diagrams, known as hard thermal loop (HTL) resummation [48], when the loop momenta are of the order of the temperature. This reorganized perturbation theory, known as HTL perturbation theory (HTLpt), leads to gauge-independent results for various physical quantities [49-65]. Within this one-loop HTLpt, the thermomagnetic correction to the quark self-energy [66], quark-gluon three-point [66] function at zero chemical potential, and four-point [67] function at finite chemical potential in the weak-field limit have been computed. The fermion self-energy has also been extended to the case of nonzero chemical potential and the pressure of a weakly magnetized QCD plasma [68] has also been obtained.

In recent years, a huge amount of activity is underway to explore the properties of a hot medium with a background magnetic field using phenomenology as well as using thermal field theory. In a thermal medium, the bulk and dynamical properties $[48,69,70]$ are characterized by the collective excitations in a timelike region and the Landau damping in a spacelike domain. The basic quantity associated with these medium properties is the two-point correlation function. In this work, we construct the general structure of the fermionic two-point functions (e.g., selfenergy and the effective propagator) in a nontrivial background such as a hot magnetized medium. We then analyze its property under the transformation of some discrete symmetries of the system, the collective fermionic spectra, QED-like three-point functions and the spectral representation of the two-point function and its consequences in a hot magnetized medium. The formulation is applicable equally well to both QED and QCD.

The paper is organized as follows; In Sec. II, the notation and set up are briefly discussed through a fermion propagator in a constant background field using Schwinger formalism. Section III has number of parts in which we obtain the general structure of the self-energy (subsection III A), the effective fermion propagator (subsection III B), the transformation properties and discrete symmetries of the effective propagator (subsection III C), the modified Dirac equations in general and for lowest Landau level (subsection III D) and the dispersion properties of the various collective modes (subsection III E) in timelike region. In Sec. IV, the general structure of the self-energy and the propagator has been verified from one-loop direct calculation. The spectral representation of the effective propagator in spacelike domain has been obtained in Sec. V. We have presented some detailed calculations for various sections and subsections in Appendix A-E. Finally, we conclude in Sec. VI.

\section{CHARGED FERMION PROPAGATOR IN BACKGROUND MAGNETIC FIELD WITHIN SCHWINGER FORMALISM}

In this section, we set the notation and briefly outline the fermionic propagator in the presence of a background magnetic field following Schwinger formalism [38]. Without any loss of generality, the background magnetic field is chosen along the $z$ direction, $\vec{B}=B \hat{z}$, and the vector potential in a symmetric gauge reads as

$$
A^{\mu}=\left(0,-\frac{y B}{2}, \frac{x B}{2}, 0\right)
$$

Below we also outline the notation we shall be using throughout: 


$$
\begin{aligned}
a^{\mu} & =\left(a^{0}, a^{1}, a^{2}, a^{3}\right)=\left(a_{0}, \vec{a}\right) ; \\
a \cdot b & \equiv a_{0} b_{0}-\vec{a} \cdot \vec{b} ; \quad g^{\mu \nu}=\operatorname{diag}(1,-1,-1,-1), \\
a^{\mu} & =a_{\|}^{\mu}+a_{\perp}^{\mu} ; \quad a_{\|}^{\mu}=\left(a^{0}, 0,0, a^{3}\right) ; \\
a_{\perp}^{\mu} & =\left(0, a^{1}, a^{2}, 0\right) \quad g^{\mu \nu}=g_{\|}^{\mu \nu}+g_{\perp}^{\mu \nu} ; \\
g_{\|}^{\mu \nu} & =\operatorname{diag}(1,0,0,-1) ; \quad g_{\perp}^{\mu \nu}=\operatorname{diag}(0,-1,-1,0), \\
(a \cdot b) & =(a \cdot b)_{\|}-(a \cdot b)_{\perp} ; \quad(a \cdot b)_{\|}=a^{0} b^{0}-a^{3} b^{3} ; \\
(a \cdot b)_{\perp} & =a^{1} b^{1}+a^{2} b^{2}, \quad \not d=\gamma^{\mu} a_{\mu}=\not \phi_{\|}+\not \phi_{\perp} ; \\
\phi_{\|} & =\gamma^{0} a_{0}-\gamma^{3} a^{3} ; \quad \not \phi_{\perp}=\gamma^{1} a^{1}+\gamma^{2} a^{2},
\end{aligned}
$$

where $\|$ and $\perp$ are, respectively, the parallel and perpendicular components, which would be separated out due to the presence of the background magnetic field.

Now, the fermionic two-point function is written as

$$
\begin{aligned}
S\left(x, x^{\prime}\right)= & -i C\left(x, x^{\prime}\right) \int_{0}^{\infty} d s \frac{1}{s \sin \left(q_{f} B s\right)} \\
& \times \exp \left(-i m_{f}^{2} s+i q_{f} B s \Sigma_{3}\right) \\
& \times \exp \left[-\frac{i}{4 s}\left(\left(x-x^{\prime}\right)_{\|}^{2}-\frac{q_{f} B s}{\tan \left(q_{f} B s\right)}\left(x-x^{\prime}\right)_{\perp}^{2}\right)\right] \\
& \times\left[m_{f}+\frac{1}{2 s}\left(\left(\not x_{\|}-\not \chi_{\|}^{\prime}\right)-\frac{q_{f} B s}{\sin \left(q_{f} B s\right)}\right.\right. \\
& \left.\left.\times \exp \left(-i q_{f} B s \Sigma_{3}\right)\left(\not \not_{\perp}-\not x_{\perp}^{\prime}\right)\right)\right],
\end{aligned}
$$

where the parameter $s$ is called Schwinger proper time variable [38]. We note that $m_{f}$ and $q_{f}$ are mass and absolute charge of the fermion of flavor $f$, respectively. The phase factor, $C\left(x, x^{\prime}\right)$, is independent of $s$ but is responsible for breaking of both gauge and translational invariance. Remaining part, denoted as $\mathcal{S}\left(x-x^{\prime}\right)$, is translationally invariant. However, as shown below, $C\left(x, x^{\prime}\right)$ drops out for a gauge invariant calculation. Now $C\left(x, x^{\prime}\right)$ reads as

$C\left(x, x^{\prime}\right)=C \exp \left[i q_{f} \int_{x^{\prime}}^{x} d \xi^{\mu}\left(A_{\mu}(\xi)+\frac{1}{2} F_{\mu \nu}\left(\xi-x^{\prime}\right)^{\nu}\right)\right]$,

where $C$ is just a number. The integral in the exponential is independent of the path taken between $x$ and $x^{\prime}$ and, choosing it as a straight line, one can write

$C\left(x, x^{\prime}\right)=C \Phi\left(x, x^{\prime}\right)=C \exp \left[i q_{f} \int_{x^{\prime}}^{x} d \xi^{\mu} A_{\mu}(\xi)\right]$.

Using the gauge transformation $A^{\mu}(\xi) \rightarrow A^{\mu}(\xi)+\partial^{\mu} \Lambda(\xi)$, and choosing the symmetric gauge as given in (1), the phase factor $\Phi\left(x, x^{\prime}\right)$ becomes 1 if we take [66]

$$
\Lambda(\xi)=\frac{B}{2}\left(x_{2}^{\prime} \xi_{1}-x_{1}^{\prime} \xi_{2}\right) .
$$

From Eq. (3), the momentum space propagator can be obtained as

$$
\begin{aligned}
S(K)= & \int d^{4} x e^{i K \cdot x} \mathcal{S}\left(x-x^{\prime}\right) \\
= & -i \int_{0}^{\infty} d s \exp \left[i s\left(K_{\|}^{2}-\frac{\tan \left(q_{f} B s\right)}{q_{f} B s} K_{\perp}^{2}-m_{f}^{2}\right)\right] \\
& \times\left[\left(1+\gamma_{1} \gamma_{2} \tan \left(q_{f} B s\right)\right)\left(K_{\|}+m_{f}\right)-\sec ^{2}\left(q_{f} B s\right) K_{\perp}\right] \\
= & \exp \left(-K_{\perp}{ }^{2} /\left|q_{f} B\right|\right) \sum_{l=0}^{\infty}(-1)^{n} \frac{D_{n}\left(q_{f} B, K\right)}{K_{\|}^{2}-m_{f}^{2}-2 l\left|q_{f} B\right|},
\end{aligned}
$$

where $k_{\perp}^{2}=2 l\left|q_{f} B\right|$, is quantized with Landau level $l=0,1, \ldots$, and

$$
\begin{aligned}
D_{l}\left(q_{f} B, K\right)= & \left(K_{\|}+m_{f}\right)\left[\left(1-i \gamma_{1} \gamma_{2}\right) L_{l}\left(2 \frac{K_{\perp}^{2}}{\left|q_{f} B\right|}\right)\right. \\
& \left.-\left(1+i \gamma_{1} \gamma_{2}\right) L_{l-1}\left(2 \frac{K_{\perp}^{2}}{\left|q_{f} B\right|}\right)\right] \\
& -4 K_{\perp} L_{l-1}^{1}\left(2 \frac{K_{\perp}^{2}}{\left|q_{f} B\right|}\right)
\end{aligned}
$$

where $L_{l}(x)$ is Laguerre polynomial, $L_{l}^{j}(x)$ is associated Laguerre polynomial with $L_{-1}^{j}(x)=0$ and both $j$ is a nonnegative integer.

Below we discuss the structure of the propagator in (7) in the presence of the background magnetic field. Since the fermion propagator is $4 \times 4$ matrix, a new matrix $\left(K_{\|}+m_{f}\right) i \gamma_{1} \gamma_{2}$ appears in addition to that of the vacuum structure $\left(\alpha^{\prime} K, \alpha^{\prime}\left(K^{2}\right)\right.$ is a Lorentz invariant structure function) for a chirally symmetric theory. One can now write the new matrix for a chirally symmetric theory in terms of background electromagnetic field tensor $F^{\rho \lambda}$ as

$$
i \gamma_{1} \gamma_{2} K_{\|} B=-\gamma_{5} K^{\mu} \tilde{F}_{\mu \nu} \gamma^{\nu}
$$

where the background dual field tensor reads as

$$
\tilde{F}_{\mu \nu}=\frac{1}{2} \epsilon_{\mu \nu \rho \lambda} F^{\rho \lambda} .
$$

The structure of a chirally symmetric free fermion propagator in the presence of only a magnetic field can be viewed as $\left(\alpha^{\prime} K+\delta^{\prime} \gamma_{5} K^{\mu} \tilde{F}_{\mu \nu} \gamma^{\nu}\right)$, where $\delta^{\prime}$ is a new structure constant that appears due to the presence of a background magnetic field. When a fermion propagates only in a hot medium, then the vacuum part will be modified only due to the thermal background [69] as $\left(\alpha^{\prime} K+\beta^{\prime} \not l\right)$, where $u$ is the 
four-velocity of the heat bath. When a fermion moves in a nontrivial background such as a hot magnetized medium, then one can write (9) as

$$
i \gamma_{1} \gamma_{2} K_{\|}=-\gamma_{5}[(K . n) \not h-(K . u) \not h]
$$

where

$$
n_{\mu}=\frac{1}{2 B} \epsilon_{\mu \nu \rho \lambda} u^{\nu} F^{\rho \lambda}=\frac{1}{B} u^{\nu} \tilde{F}_{\mu \nu}
$$

and the four-velocity in the rest frame of the heat bath and the direction of the magnetic field $B$, respectively, given as

$$
\begin{aligned}
& u^{\mu}=(1,0,0,0), \\
& n^{\mu}=(0,0,0,1) .
\end{aligned}
$$

One can notice that in a hot magnetized medium both $u$ and $n$ are correlated as given in (12) and the contribution due to magnetic field in (9) in the presence of a heat bath becomes a thermomagnetic contribution. We also further note that in the absence of a heat bath, (11) reduces to (9), which is not obvious by inspection but we would see later.

\section{GENERAL STRUCTURE OF FERMION TWO-POINT FUNCTION IN A HOT MAGNETIZED MEDIUM}

In the previous section, the modification of a free propagator has been discussed briefly in the presence of a background magnetic field. In this section, we would like to obtain the most general structure of a fermion selfenergy, the effective fermion propagator and some of its properties in a nontrivial background such as a hot magnetized medium. We would also discuss the modified Dirac equation and the fermion dispersion spectrum in a hot magnetized medium. For a thermal bath, we would use HTL approximation and any other approximation required for the purpose will be stated therein.

\section{A. General structure of the fermion self-energy}

The fermionic self-energy is a matrix as well as a Lorentz scalar. However, in the presence of a nontrivial background, e.g., heat bath and magnetic field, the boost and rotational symmetries of the system are broken. The general structure of fermion self-energy for hot magnetized medium can be written by the following arguments. The self-energy $\Sigma(P)$ is a $4 \times 4$ matrix which depends, in present case, on the four-momentum of the fermion $P$, the velocity of the heat bath $u$ and the direction of the magnetic field $n$. Now, any $4 \times 4$ matrix can be expanded in terms of 16 basis matrices: $\left\{\mathbb{1}, \gamma_{5}, \gamma_{\mu}, \gamma_{\mu} \gamma_{5}, \sigma_{\mu \nu}\right\}$, which are the unit matrix, the four $\gamma$ matrices, the six $\sigma_{\mu \nu}$ matrices, the four $\gamma_{5} \gamma_{\mu}$ matrices and finally $\gamma_{5}$. So, the general structure can be written as

$$
\begin{aligned}
\Sigma(P)= & -\alpha \mathbb{1}-\beta \gamma_{5}-a \not P-b \not h-c \not h-a^{\prime} \gamma_{5} \not P-b^{\prime} \gamma_{5} \not h-c^{\prime} \gamma_{5} \not h \\
& -h \sigma_{\mu \nu} P^{\mu} P^{\nu}-h^{\prime} \sigma_{\mu \nu} u^{\mu} u^{\nu}-\kappa \sigma_{\mu \nu} n^{\mu} n^{\nu}-d \sigma_{\mu \nu} P^{\mu} u^{\nu} \\
& -d^{\prime} \sigma_{\mu \nu} n^{\mu} P^{\nu}-\kappa^{\prime} \sigma_{\mu \nu} u^{\mu} n^{\nu},
\end{aligned}
$$

where various coefficients are known as structure functions. We note that the combinations involving $\sigma_{\mu \nu}$ do not appear due to antisymmetric nature of it in any loop order of selfenergy. Also in a chirally invariant theory, the terms $\alpha \rrbracket$ and $\gamma_{5} \beta$ will not appear as they would break the chiral symmetry. The term $\gamma_{5} \not \mathcal{~ w o u l d ~ a p p e a r ~ i n ~ t h e ~ s e l f - e n e r g y ~}$ if fermions interact with an axial vector. ${ }^{1}$ By dropping those in (14) for chirally symmetric theory, one can now write

$$
\Sigma(P)=-a \not P-b \not h-c \not h-b^{\prime} \gamma_{5} \not h-c^{\prime} \gamma_{5} \not h .
$$

Now we point out that some important information is encoded into the fermion propagator in (7) through (11) for a hot magnetized medium. This suggests that $c$ h should not appear in the fermion self-energy ${ }^{2}$ and the most general form of the fermion self-energy for a hot magnetized medium becomes

$$
\Sigma(P)=-a \not P-b \not h-b^{\prime} \gamma_{5} \not h-c^{\prime} \gamma_{5} \not h
$$

When a fermion propagates in a vacuum, then $b=b^{\prime}=$ $c^{\prime}=0$ and $\Sigma(P)=-a \not P$. But when it propagates in a background of pure magnetic field without any heat bath, then $a \neq 0, b=0$ and the structure functions, $b^{\prime}$ and $c^{\prime}$, will depend only on the background magnetic field as we will see later. When a fermion propagates in a heat bath, then $a \neq 0, b \neq 0$ but both $b^{\prime}$ and $c^{\prime}$ vanish because there would not be any thermomagnetic corrections as can also be seen later.

We now write down the right chiral projection operator, $\mathcal{P}_{+}$and the left chiral projection operator $\mathcal{P}_{-}$, respectively, defined as:

$$
\begin{aligned}
& \mathcal{P}_{+}=\frac{1}{2}\left(\mathbb{1}+\gamma_{5}\right), \\
& \mathcal{P}_{-}=\frac{1}{2}\left(\mathbb{1}-\gamma_{5}\right),
\end{aligned}
$$

\footnotetext{
${ }^{1}$ The presence of an axial gauge coupling leads to chiral or axial anomaly and a chirally invariant theory does not allow this. Other way, the preservation of both chiral and axial symmetries is impossible, a choice must be made which one should be preserved. For a chirally invariant theory, this term drops out. Also the presence of $\gamma_{5}$ in a Lagrangian violates parity invariance.

${ }^{2}$ We have checked that even if one keeps $c \not h$, the coefficient $c$ becomes zero in one-loop order in the weak-field approximation.
} 
which satisfy the usual properties of projection operator:

$$
\begin{aligned}
& \mathcal{P}_{ \pm}^{2}=\mathcal{P}_{ \pm}, \quad \mathcal{P}_{+} \mathcal{P}_{-}=\mathcal{P}_{-} \mathcal{P}_{+}=0, \\
& \mathcal{P}_{+}+\mathcal{P}_{-}=1, \quad \mathcal{P}_{+}-\mathcal{P}_{-}=\gamma_{5} .
\end{aligned}
$$

Using the chirality projection operators, the general structure of the self-energy in (16) can be casted in the following form

$$
\Sigma(P)=-\mathcal{P}_{+} \not \mathcal{P}_{-}-\mathcal{P}_{-} D \mathcal{P}_{+},
$$

where $\not \subset$ and $\not D$ are defined as

$$
\begin{aligned}
& \boldsymbol{C}=a P+\left(b+b^{\prime}\right) \not h+c^{\prime} \not h, \\
& D=a \not P+\left(b-b^{\prime}\right) \not h-c^{\prime} \not h .
\end{aligned}
$$

From (16) one obtains the general form of the various structure functions as

$$
\begin{aligned}
a & =\frac{1}{4} \frac{\operatorname{Tr}(\Sigma \not \supset)-(P . u) \operatorname{Tr}(\Sigma \not /)}{(P . u)^{2}-P^{2}}, \\
b & =\frac{1}{4} \frac{-(P . u) \operatorname{Tr}(\Sigma \not \supset)+P^{2} \operatorname{Tr}(\Sigma \not \iota)}{(P . u)^{2}-P^{2}}, \\
b^{\prime} & =-\frac{1}{4} \operatorname{Tr}\left(\not / \Sigma \gamma_{5}\right), \\
c^{\prime} & =\frac{1}{4} \operatorname{Tr}\left(\not h \Sigma \gamma_{5}\right),
\end{aligned}
$$

which are also Lorentz scalars. Beside $T$ and $B$, they would also depend on three Lorentz scalars defined by

$$
\begin{aligned}
\omega & \equiv P^{\mu} u_{\mu}, \\
p^{3} & \equiv-P^{\mu} n_{\mu}=p_{z}, \\
p_{\perp} & \equiv\left[\left(P^{\mu} u_{\mu}\right)^{2}-\left(P^{\mu} n_{\mu}\right)^{2}-\left(P^{\mu} P_{\mu}\right)\right]^{1 / 2} .
\end{aligned}
$$

Since $P^{2}=\omega^{2}-p_{\perp}^{2}-p_{z}{ }^{2}$, we may interpret $\omega, p_{\perp}, p_{z}$ as Lorentz invariant energy, transverse momentum, longitudinal momentum respectively. All these structure functions for one-loop order in a weak field and HTL approximations have been computed in Appendix A and quoted here ${ }^{3}$ as

\footnotetext{
${ }^{3}$ In the weak-field approximation, the domain of applicability becomes $m_{t h}^{2}\left(\sim g^{2} T^{2}\right)<q_{f} B<T^{2}$ instead of $m^{2}<q_{f} B<T^{2}$ as discussed in Appendix A.
}

$$
\begin{aligned}
& a\left(p_{0},|\vec{p}|\right)=-\frac{m_{\text {th }}^{2}}{|\vec{p}|^{2}} Q_{1}\left(\frac{p_{0}}{|\vec{p}|}\right), \\
& b\left(p_{0},|\vec{p}|\right)=\frac{m_{t h}^{2}}{|\vec{p}|}\left[\frac{p_{0}}{|\vec{p}|} Q_{1}\left(\frac{p_{0}}{|\vec{p}|}\right)-Q_{0}\left(\frac{p_{0}}{|\vec{p}|}\right)\right],
\end{aligned}
$$$$
b^{\prime}\left(p_{0},|\vec{p}|\right)=4 C_{F} g^{2} M^{2}\left(T, m_{f}, q_{f} B\right) \frac{p_{z}}{|\vec{p}|^{2}} Q_{1}\left(\frac{p_{0}}{|\vec{p}|}\right),
$$

$c^{\prime}\left(p_{0},|\vec{p}|\right)=4 C_{F} g^{2} M^{2}\left(T, m_{f}, q_{f} B\right) \frac{1}{|\vec{p}|} Q_{0}\left(\frac{p_{0}}{|\vec{p}|}\right)$.

We note that the respective vacuum contributions in $a, b^{\prime}$ and $c^{\prime}$ have been dropped by the choice of the renormalization prescription, and the general structure of the selfenergy, as found in Appendix A, agrees with that in (16).

\section{B. Effective fermion propagator}

The effective fermion propagator is given by DysonSchwinger equation (see Fig. 1) which reads as

$$
S^{*}(P)=\frac{1}{P-\Sigma(P)},
$$

and the inverse fermion propagator reads as

$$
S^{*-1}(P)=P-\Sigma(P) .
$$

Using (19) the inverse fermion propagator can be written as

$$
\begin{aligned}
S^{*-1}(P)= & \mathcal{P}_{+}\left[\left(1+a\left(p_{0},|\vec{p}|\right)\right) \not P+\left(b\left(p_{0},|\vec{p}|\right)\right.\right. \\
& \left.\left.+b^{\prime}\left(p_{0}, p_{\perp}, p_{z}\right)\right) \not h+c^{\prime}\left(p_{0},|\vec{p}|\right) \not h\right] \mathcal{P}_{-} \\
& +\mathcal{P}_{-}\left[\left(1+a\left(p_{0},|\vec{p}|\right)\right) P+\left(b\left(p_{0},|\vec{p}|\right)\right.\right. \\
& \left.\left.-b^{\prime}\left(p_{0}, p_{\perp}, p_{z}\right)\right) \not h-c^{\prime}\left(p_{0},|\vec{p}|\right) \not h\right] \mathcal{P}_{+} \\
= & \mathcal{P}_{+} \not \mathcal{P}_{-}+\mathcal{P}_{-} \not R \mathcal{P}_{+},
\end{aligned}
$$

where $\not L$ and $\not R$ can be obtained from two four-vectors given by

$$
\begin{aligned}
L^{\mu}\left(p_{0}, p_{\perp}, p_{z}\right)= & \mathcal{A}\left(p_{0},|\vec{p}|\right) P^{\mu}+\mathcal{B}_{+}\left(p_{0}, p_{\perp}, p_{z}\right) u^{\mu} \\
& +c^{\prime}\left(p_{0},|\vec{p}|\right) n^{\mu}, \\
R^{\mu}\left(p_{0}, p_{\perp}, p_{z}\right)= & \mathcal{A}\left(p_{0},|\vec{p}|\right) P^{\mu}+\mathcal{B}_{-}\left(p_{0}, p_{\perp}, p_{z}\right) u^{\mu} \\
& -c^{\prime}\left(p_{0},|\vec{p}|\right) n^{\mu},
\end{aligned}
$$

with 


$$
\begin{gathered}
\mathcal{A}\left(p_{0},|\vec{p}|\right)=1+a\left(p_{0},|\vec{p}|\right) \\
\mathcal{B}_{ \pm}\left(p_{0}, p_{\perp}, p_{z}\right)=b\left(p_{0},|\vec{p}|\right) \pm b^{\prime}\left(p_{0}, p_{\perp}, p_{z}\right) .
\end{gathered}
$$

Using (26) in (24), the propagator can now be written as

$$
S^{*}(P)=\mathcal{P}_{-} \frac{\not L}{L^{2}} \mathcal{P}_{+}+\mathcal{P}_{+} \frac{\not R}{R^{2}} \mathcal{P}_{-},
$$

where we have used the properties of the projection operators $\mathcal{P}_{ \pm} \gamma^{\mu}=\gamma^{\mu} \mathcal{P}_{\mp}, \mathcal{P}_{ \pm}^{2}=\mathcal{P}_{ \pm}$, and $\mathcal{P}_{+} \mathcal{P}_{-}=\mathcal{P}_{-} \mathcal{P}_{+}=0$. It can be checked that $S^{*}(P) S^{*-1}(P)=\mathcal{P}_{+}+\mathcal{P}_{-}=\mathbb{1}$. Also we have

$$
\begin{aligned}
L^{2}=L^{\mu} L_{\mu} & =\left(\mathcal{A} p_{0}+\mathcal{B}_{+}\right)^{2}-\left[\left(\mathcal{A} p_{z}+c^{\prime}\right)^{2}+\mathcal{A}^{2} p_{\perp}^{2}\right] \\
& =L_{0}^{2}-|\vec{L}|^{2}, \\
R^{2}=R^{\mu} R_{\mu} & =\left(\mathcal{A} p_{0}+\mathcal{B}_{-}\right)^{2}-\left[\left(\mathcal{A} p_{z}-c^{\prime}\right)^{2}+\mathcal{A}^{2} p_{\perp}^{2}\right] \\
& =R_{0}^{2}-|\vec{R}|^{2},
\end{aligned}
$$

where we have used $u^{2}=1, n^{2}=-1, u \cdot n=0$, $P \cdot u=p_{0}$, and $P \cdot n=-p_{z}$. Note that we have suppressed the functional dependencies of $L, R, \mathcal{A}, \mathcal{B}_{ \pm}$and $c^{\prime}$ and would bring them back whenever necessary.

For the lowest Landau level (LLL), $l=0 \Rightarrow p_{\perp}=0$, and these relations reduce to

$$
\begin{aligned}
& L_{L L L}^{2}=\left(\mathcal{A} p_{0}+\mathcal{B}_{+}\right)^{2}-\left(\mathcal{A} p_{z}+c^{\prime}\right)^{2}=L_{0}^{2}-L_{z}^{2}, \\
& R_{L L L}^{2}=\left(\mathcal{A} p_{0}+\mathcal{B}_{-}\right)^{2}-\left(\mathcal{A} p_{z}-c^{\prime}\right)^{2}=R_{0}^{2}-R_{z}^{2} .
\end{aligned}
$$

The poles of the effective propagator, $L^{2}=0$ and $R^{2}=0$, give rise to quasi-particle dispersion relations in a hot magnetized medium. There will be four collective modes with positive energies: two from $L^{2}=0$ and two from $R^{2}=0$. Nevertheless, we will discuss dispersion properties later.

\section{Transformation properties of structure functions and propagators}

First, we outline some transformation properties of the various structure functions as obtained in (23a), (23b), (23c) and (23d).

(1) Under the transformation $\vec{p} \rightarrow-\vec{p}=\left(p_{\perp},-p_{z}\right)$,

$$
\begin{aligned}
a\left(p_{0},|-\vec{p}|\right) & =a\left(p_{0},|\vec{p}|\right), \\
b\left(p_{0},|-\vec{p}|\right) & =b\left(p_{0},|\vec{p}|\right), \\
b^{\prime}\left(p_{0}, p_{\perp},-p_{z}\right) & =-b^{\prime}\left(p_{0}, p_{\perp}, p_{z}\right), \\
c^{\prime}\left(p_{0},|-\vec{p}|\right) & =c^{\prime}\left(p_{0},|\vec{p}|\right) .
\end{aligned}
$$

(2) For $p_{0} \rightarrow-p_{0}$ :

$$
\begin{aligned}
a\left(-p_{0},|\vec{p}|\right) & =a\left(p_{0},|\vec{p}|\right), \\
b\left(-p_{0},|\vec{p}|\right) & =-b\left(p_{0},|\vec{p}|\right), \\
b^{\prime}\left(-p_{0}, p_{\perp}, p_{z}\right) & =b^{\prime}\left(p_{0}, p_{\perp}, p_{z}\right), \\
c^{\prime}\left(-p_{0},|\vec{p}|\right) & =-c^{\prime}\left(p_{0},|\vec{p}|\right) .
\end{aligned}
$$

(3) For $P \rightarrow-P=\left(-p_{0},-\vec{p}\right)$ :

$$
\begin{aligned}
a\left(-p_{0},|-\vec{p}|\right) & =a\left(p_{0},|\vec{p}|\right), \\
b\left(-p_{0},|-\vec{p}|\right) & =-b\left(p_{0},|\vec{p}|\right), \\
b^{\prime}\left(-p_{0}, p_{\perp},-p_{z}\right) & =-b^{\prime}\left(p_{0}, p_{\perp}, p_{z}\right), \\
c^{\prime}\left(-p_{0},|-\vec{p}|\right) & =-c^{\prime}\left(p_{0},|\vec{p}|\right) .
\end{aligned}
$$

We have used the fact that $Q_{0}(-x)=-Q_{0}(x)$ and $Q_{1}(-x)=Q_{1}(x)$.

Now based on the above, we also note the transformation properties of those quantities appearing in the propagator:

(1) For $\mathcal{A}$ :

$$
\begin{gathered}
\mathcal{A}\left(p_{0}, p_{\perp}, p_{z}\right) \stackrel{\vec{p} \rightarrow-\vec{p}}{\longrightarrow} \mathcal{A}\left(p_{0}, p_{\perp}, p_{z}\right), \\
\mathcal{A}\left(p_{0}, p_{\perp}, p_{z}\right) \stackrel{p_{0} \rightarrow-p_{0}}{\longrightarrow} \mathcal{A}\left(p_{0}, p_{\perp}, p_{z}\right), \\
\mathcal{A}\left(p_{0}, p_{\perp}, p_{z}\right) \stackrel{\substack{p_{0} \rightarrow-p_{0} \\
\vec{p} \rightarrow-\vec{p}}}{\longrightarrow} \mathcal{A}\left(p_{0}, p_{\perp}, p_{z}\right) .
\end{gathered}
$$

(2) For $\mathcal{B}_{ \pm}$:

$$
\begin{aligned}
& \mathcal{B}_{ \pm}\left(p_{0}, p_{\perp}, p_{z}\right) \stackrel{\vec{p} \rightarrow-\vec{p}}{\longrightarrow} \mathcal{B}_{\mp}\left(p_{0}, p_{\perp}, p_{z}\right), \\
& \mathcal{B}_{ \pm}\left(p_{0}, p_{\perp}, p_{z}\right) \stackrel{p_{0} \rightarrow-p_{0}}{\longrightarrow}-\mathcal{B}_{\mp}\left(p_{0}, p_{\perp}, p_{z}\right), \\
& \mathcal{B}_{ \pm}\left(p_{0}, p_{\perp}, p_{z}\right) \stackrel{p_{0} \rightarrow-p_{0}}{\stackrel{\vec{p} \rightarrow-\vec{p}}{\longrightarrow}}-\mathcal{B}_{ \pm}\left(p_{0}, p_{\perp}, p_{z}\right) .
\end{aligned}
$$

Using the above transformation properties, it can be shown that $\not, R, L^{2}$ and $R^{2}$, respectively given in (27a), (27b), (30a) and (30b) transform as

$$
\begin{aligned}
& \not\left(p_{0}, p_{\perp}, p_{z}\right) \stackrel{\vec{p} \rightarrow-\vec{p}}{\longrightarrow} \mathcal{A}\left(p_{0},|\vec{p}|\right)\left(p_{0} \gamma^{0}+\vec{p} \cdot \vec{\gamma}\right) \\
& +\mathcal{B}_{-}\left(p_{0}, p_{\perp}, p_{z}\right) \not \iota+c^{\prime}\left(p_{0},|\vec{p}|\right) \not h, \\
& \not R\left(p_{0}, p_{\perp}, p_{z}\right) \stackrel{\vec{p} \rightarrow-\vec{p}}{\longrightarrow} \mathcal{A}\left(p_{0},|\vec{p}|\right)\left(p_{0} \gamma^{0}+\vec{p} \cdot \vec{\gamma}\right) \\
& +\mathcal{B}_{+}\left(p_{0}, p_{\perp}, p_{z}\right) \not h-c^{\prime}\left(p_{0},|\vec{p}|\right) \not h, \\
& L^{2}\left(p_{0}, p_{\perp}, p_{z}\right) \stackrel{\vec{p} \rightarrow-\vec{p}}{\longrightarrow} R^{2}\left(p_{0}, p_{\perp}, p_{z}\right), \\
& R^{2}\left(p_{0}, p_{\perp}, p_{z}\right) \stackrel{\vec{p} \rightarrow-\vec{p}}{\longrightarrow} L^{2}\left(p_{0}, p_{\perp}, p_{z}\right),
\end{aligned}
$$


and

$$
\begin{aligned}
& \not L\left(p_{0}, p_{\perp}, p_{z}\right) \underset{\vec{p} \rightarrow-\vec{p}}{\stackrel{p_{0} \rightarrow-p_{0}}{\longrightarrow}}-\not L\left(p_{0}, p_{\perp}, p_{z}\right), \\
& \not R\left(p_{0}, p_{\perp}, p_{z}\right) \underset{\vec{p} \rightarrow-\vec{p}}{\stackrel{p_{0} \rightarrow-p_{0}}{\longrightarrow}}-\not R\left(p_{0}, p_{\perp}, p_{z}\right), \\
& L^{2}\left(p_{0}, p_{\perp}, p_{z}\right) \underset{\vec{p} \rightarrow-\vec{p}}{\stackrel{p_{0} \rightarrow-p_{0}}{\longrightarrow}} L^{2}\left(p_{0}, p_{\perp}, p_{z}\right), \\
& R^{2}\left(p_{0}, p_{\perp}, p_{z}\right) \underset{\vec{p} \rightarrow-\vec{p}}{\stackrel{p_{0} \rightarrow-p_{0}}{\longrightarrow}} R^{2}\left(p_{0}, p_{\perp}, p_{z}\right) .
\end{aligned}
$$

Now we are in a position to check the transformation properties of the effective propagator under some of the discrete symmetries:

\section{Chirality}

Under chirality the fermion propagator transform as [71]

$$
S\left(p_{0}, \vec{p}\right) \rightarrow-\gamma_{5} S\left(p_{0}, \vec{p}\right) \gamma_{5} .
$$

The effective propagator, $S^{*}\left(p_{0}, p_{\perp}, p_{z}\right)$, in (29) transforms under chirality as

$$
\begin{aligned}
- & \gamma_{5} S^{*}\left(p_{0}, p_{\perp}, p_{z}\right) \gamma_{5} \\
= & -\gamma_{5} \mathcal{P}_{-} \frac{\not L\left(p_{0}, p_{\perp}, p_{z}\right)}{L^{2}\left(p_{0}, p_{\perp}, p_{z}\right)} \mathcal{P}_{+} \gamma_{5} \\
& -\gamma_{5} \mathcal{P}_{+} \frac{\not R\left(p_{0}, p_{\perp}, p_{z}\right)}{R^{2}\left(p_{0}, p_{\perp}, p_{z}\right)} \mathcal{P}_{-} \gamma_{5} \\
= & \mathcal{P}_{+} \frac{\not L\left(p_{0}, p_{\perp}, p_{z}\right)}{L^{2}\left(p_{0}, p_{\perp}, p_{z}\right)} \mathcal{P}_{+}+\mathcal{P}_{-} \frac{\not R\left(p_{0}, p_{\perp}, p_{z}\right)}{R^{2}\left(p_{0}, p_{\perp}, p_{z}\right)} \mathcal{P}_{-} \\
= & S^{*}\left(p_{0}, p_{\perp}, p_{z}\right),
\end{aligned}
$$

which satisfies (39) and indicates that it is chirally invariant.

\section{Reflection}

Under reflection the fermion propagator transforms [71] as

$$
S\left(p_{0}, \vec{p}\right) \rightarrow S\left(p_{0},-\vec{p}\right) .
$$

The effective propagator, $S^{*}\left(p_{0}, p_{\perp}, p_{z}\right)$, in (29) transforms under reflection as

$$
\begin{aligned}
S^{*}\left(p_{0}, p_{\perp},-p_{z}\right)= & \mathcal{P}_{-} \frac{\not L\left(p_{0}, p_{\perp},-p_{z}\right)}{L^{2}\left(p_{0}, p_{\perp},-p_{z}\right)} \mathcal{P}_{+}+\mathcal{P}_{+} \frac{\not R\left(p_{0}, p_{\perp},-p_{z}\right)}{R^{2}\left(p_{0}, p_{\perp},-p_{z}\right)} \mathcal{P}_{-} \\
= & \mathcal{P}_{-} \frac{\mathcal{A}\left(p_{0},|\vec{p}|\right)\left(p_{0} \gamma^{0}+\vec{p} \cdot \vec{\gamma}\right)+\mathcal{B}_{-}\left(p_{0}, p_{\perp}, p_{z}\right) \not h+c^{\prime}\left(p_{0},|\vec{p}|\right) \hbar h}{R^{2}\left(p_{0}, p_{\perp}, p_{z}\right)} \mathcal{P}_{+} \\
& +\mathcal{P}_{+} \frac{\mathcal{A}\left(p_{0},|\vec{p}|\right)\left(p_{0} \gamma^{0}+\vec{p} \cdot \vec{\gamma}\right)+\mathcal{B}_{+}\left(p_{0}, p_{\perp}, p_{z}\right) \not h-c^{\prime}\left(p_{0},|\vec{p}|\right) \not h}{L^{2}\left(p_{0}, p_{\perp}, p_{z}\right)} \mathcal{P}_{-} \\
\neq & S^{*}\left(p_{0}, p_{\perp}, p_{z}\right) .
\end{aligned}
$$

However, now considering the rest frame of the heat bath, $u^{\mu}=(1,0,0,0)$, and the background magnetic field along $z$-direction, $n^{\mu}=(0,0,0,1)$, one can write $(42)$ as

$$
\begin{aligned}
S^{*}\left(p_{0}, p_{\perp},-p_{z}\right)= & \mathcal{P}_{-} \frac{\mathcal{A}\left(p_{0},|\vec{p}|\right)\left(p_{0} \gamma^{0}+\vec{p} \cdot \vec{\gamma}\right)+\mathcal{B}_{-}\left(p_{0}, p_{\perp}, p_{z}\right) \gamma_{0}-c^{\prime}\left(p_{0},|\vec{p}|\right) \gamma^{3}}{R^{2}\left(p_{0}, p_{\perp}, p_{z}\right)} \mathcal{P}_{+} \\
& +\mathcal{P}_{+} \frac{\mathcal{A}\left(p_{0},|\vec{p}|\right)\left(p_{0} \gamma^{0}+\vec{p} \cdot \vec{\gamma}\right)+\mathcal{B}_{+}\left(p_{0}, p_{\perp}, p_{z}\right) \gamma_{0}+c^{\prime}\left(p_{0},|\vec{p}|\right) \gamma^{3}}{L^{2}\left(p_{0}, p_{\perp}, p_{z}\right)} \mathcal{P}_{-} \\
\neq & S^{*}\left(p_{0}, p_{\perp}, p_{z}\right) .
\end{aligned}
$$

As seen in both cases the reflection symmetry is violated as we will see later while discussing the dispersion property of a fermion.

\section{Parity}

Under parity a fermion propagator transforms [71] as

$$
S\left(p_{0}, \vec{p}\right) \rightarrow \gamma_{0} S\left(p_{0},-\vec{p}\right) \gamma_{0}
$$

The effective propagator, $S^{*}\left(p_{0}, p_{\perp}, p_{z}\right)$, in (29) under parity transforms as 


$$
\begin{aligned}
\gamma_{0} & S^{*}\left(p_{0}, p_{\perp},-p_{z}\right) \gamma_{0} \\
= & \gamma_{0} \mathcal{P}_{-} \frac{\not L\left(p_{0}, p_{\perp},-p_{z}\right)}{L^{2}\left(p_{0}, p_{\perp},-p_{z}\right)} \mathcal{P}_{+} \gamma_{0} \\
& +\gamma_{0} \mathcal{P}_{+} \frac{\not R\left(p_{0}, p_{\perp},-p_{z}\right)}{R^{2}\left(p_{0}, p_{\perp},-p_{z}\right)} \mathcal{P}_{-} \gamma_{0} \\
& =\mathcal{P}_{+} \gamma_{0} \frac{\not L\left(p_{0}, p_{\perp},-p_{z}\right)}{R^{2}\left(p_{0}, p_{\perp}, p_{z}\right)} \gamma_{0} \mathcal{P}_{-}+\mathcal{P}_{-} \gamma_{0} \frac{\not R\left(p_{0}, p_{\perp},-p_{z}\right)}{L^{2}\left(p_{0}, p_{\perp}, p_{z}\right)} \gamma_{0} \mathcal{P}_{+} \\
& \neq S^{*}\left(p_{0}, p_{\perp}, p_{z}\right),
\end{aligned}
$$

which does not obey (44), indicating that the effective propagator in general frame of reference is not parity invariant due to the background medium.

However, now considering the rest frame of the heat bath, $u^{\mu}=(1,0,0,0)$, and the background magnetic field along $z$-direction, $n^{\mu}=(0,0,0,1)$, one can write (45) by using (37a), (37b) and $\gamma_{0} \gamma^{i}=-\gamma^{i} \gamma_{0}$ as

$$
\begin{aligned}
\gamma_{0} & S^{*}\left(p_{0}, p_{\perp},-p_{z}\right) \gamma_{0} \\
& =\mathcal{P}_{+} \frac{\not R\left(p_{0}, p_{\perp}, p_{z}\right)}{R^{2}\left(p_{0}, p_{\perp}, p_{z}\right)} \mathcal{P}_{-}+\mathcal{P}_{-} \frac{\not L\left(p_{0}, p_{\perp}, p_{z}\right)}{L^{2}\left(p_{0}, p_{\perp}, p_{z}\right)} \mathcal{P}_{+} \\
& =S^{*}\left(p_{0}, p_{\perp}, p_{z}\right),
\end{aligned}
$$

which indicates that the propagator is parity invariant in the rest frame of the magnetized heat bath. We note that other discrete symmetries can also be checked but leave them on the readers.

\section{Modified Dirac equation}

\section{General case}

The effective propagator that satisfy the modified Dirac equation with spinor $U$ is given by

$$
\left(\mathcal{P}_{+} \not L \mathcal{P}_{-}+\mathcal{P}_{-} \not \mathcal{R} \mathcal{P}_{+}\right) U=0
$$

Using the chiral basis,

$$
\begin{array}{ll}
\gamma_{0}=\left(\begin{array}{ll}
0 & \mathbb{1} \\
\mathbb{1} & 0
\end{array}\right), & \vec{\gamma}=\left(\begin{array}{cc}
0 & \vec{\sigma} \\
-\vec{\sigma} & 0
\end{array}\right), \\
\gamma_{5}=\left(\begin{array}{cc}
-\mathbb{1} & 0 \\
0 & \mathbb{1}
\end{array}\right), & U=\left(\begin{array}{l}
\psi_{L} \\
\psi_{R}
\end{array}\right),
\end{array}
$$

one can write (47) as

$$
\left(\begin{array}{cc}
0 & \sigma \cdot R \\
\bar{\sigma} \cdot L & 0
\end{array}\right)\left(\begin{array}{l}
\psi_{L} \\
\psi_{R}
\end{array}\right)=0
$$

where $\psi_{R}$ and $\psi_{L}$ are two component Dirac spinors with $\sigma \equiv(1, \vec{\sigma})$ and $\bar{\sigma} \equiv(1,-\vec{\sigma})$, respectively. One can obtain nontrivial solutions with the condition

$$
\begin{aligned}
\operatorname{det}\left(\begin{array}{cc}
0 & \sigma \cdot R \\
\bar{\sigma} \cdot L & 0
\end{array}\right) & =0 \\
\operatorname{det}[L \cdot \bar{\sigma}] \operatorname{det}[R \cdot \sigma] & =0 \\
L^{2} R^{2} & =0 .
\end{aligned}
$$

We note that for a given $p_{0}(=\omega)$, either $L^{2}=0$, or $R^{2}=0$, but not both of them are simultaneously zero. This implies that i) when $L^{2}=0, \psi_{R}=0$; ii) when $R^{2}=0, \psi_{L}=0$. These dispersion conditions are same as obtained from the poles of the effective propagator in (29) as obtained in subsection III B.

(1) For $R^{2}=0$ but $L^{2} \neq 0$, the right chiral equation is given by

$$
(R \cdot \sigma) \psi_{R}=0 .
$$

Again $R^{2}=0 \Rightarrow R_{0}= \pm|\vec{R}|= \pm \sqrt{R_{x}^{2}+R_{y}^{2}+R_{z}^{2}}$ and the corresponding dispersive modes are denoted by $R^{( \pm)}$. So the solutions of (51) are

(i) $R_{0}=|\vec{R}| ; \quad$ mode $R^{(+)}$;

$$
U_{R^{(+)}}=\sqrt{\frac{|\vec{R}|+R_{z}}{2|\vec{R}|}}\left(\begin{array}{c}
0 \\
0 \\
1 \\
\frac{R_{x}+i R_{y}}{|\vec{R}|+R_{z}}
\end{array}\right)=\left(\begin{array}{c}
0 \\
\psi_{R}^{(+)}
\end{array}\right),
$$

(ii) $R_{0}=-|\vec{R}| ; \quad$ mode $R^{(-)}$;

$$
U_{R^{(-)}}=-\sqrt{\frac{|\vec{R}|+R_{z}}{2|\vec{R}|}}\left(\begin{array}{c}
0 \\
0 \\
\frac{R_{x}-i R_{y}}{|\vec{R}|+R_{z}} \\
-1
\end{array}\right)=\left(\begin{array}{c}
0 \\
\psi_{R}^{(-)}
\end{array}\right) .
$$

(2) For $L^{2}=0$ but $R^{2} \neq 0$, the left chiral equation is given by

$$
(L \cdot \bar{\sigma}) \psi_{L}=0,
$$

where $L^{2}=0$ implies two conditions; $L_{0}= \pm|\vec{L}|=$ $\pm \sqrt{L_{x}^{2}+L_{y}^{2}+L_{z}^{2}}$ and the corresponding dispersive modes are denoted by $L^{( \pm)}$. The two solutions of (53) are obtained as 
(i) $L_{0}=|\vec{L}| ; \operatorname{mode} L^{(+)}$;

$U_{L^{(+)}}=-\sqrt{\frac{|\vec{L}|+L_{z}}{2|\vec{L}|}}\left(\begin{array}{c}\frac{L_{x}-i L_{y}}{|\vec{L}|+L_{z}} \\ -1 \\ 0 \\ 0\end{array}\right)=\left(\begin{array}{c}\psi_{L}^{(+)} \\ 0\end{array}\right)$,

(i) $L_{0}=-|\vec{L}| ; \quad$ mode $L^{(-)}$;

$$
U_{L^{(-)}}=\sqrt{\frac{|\vec{L}|+L_{z}}{2|\vec{L}|}}\left(\begin{array}{c}
1 \\
\frac{L_{x}+i L_{y}}{|\vec{L}|+L_{z}} \\
0 \\
0
\end{array}\right)=\left(\begin{array}{c}
\psi_{L}^{(-)} \\
0
\end{array}\right) .
$$

We note here that $\psi_{L}^{( \pm)}$and $\psi_{R}^{( \pm)}$are only chiral eigenstates but neither the spin nor the helicity eigenstates.

\section{For lowest Landau level (LLL)}

(1) $R_{L L L}^{2}=0$ in (31b) indicates that $R_{0}= \pm R_{z}$, $R_{x}=R_{y}=0$. The two solutions obtained, respectively, in (B5) and (B6) in Appendix B are given as

(i) $R_{0}=R_{z} ; \quad$ mode $R^{(+)}$;

$$
U_{R^{(+)}}=\left(\begin{array}{l}
0 \\
0 \\
1 \\
0
\end{array}\right)=\left(\begin{array}{c}
0 \\
\chi_{+}
\end{array}\right) \text {. }
$$

(ii) $R_{0}=-R_{z} ; \quad$ mode $R^{(-)}$;

$$
U_{R^{(-)}}=\left(\begin{array}{l}
0 \\
0 \\
0 \\
1
\end{array}\right)=\left(\begin{array}{c}
0 \\
\chi_{-}
\end{array}\right) \text {, }
$$

where $\chi_{+}=\left(\begin{array}{l}1 \\ 0\end{array}\right)$ and $\chi_{-}=\left(\begin{array}{l}0 \\ 1\end{array}\right)$.

(2) For LLL, $L_{L L L}^{2}=0$ in (31a) indicates that $L_{0}= \pm L_{z}, L_{x}=L_{y}=0$. The two solutions obtained, respectively, in (B7) and (B8) in Appendix B are given as

(i) $L_{0}=L_{z} ; \quad$ mode $L^{(+)}$;

$$
U_{L^{(+)}}=\left(\begin{array}{l}
0 \\
1 \\
0 \\
0
\end{array}\right)=\left(\begin{array}{c}
\chi_{-} \\
0
\end{array}\right) \text {, }
$$

(i) $L_{0}=-L_{z} ; \quad$ mode $L^{(-)}$;

$$
U_{L^{(-)}}=\left(\begin{array}{l}
1 \\
0 \\
0 \\
0
\end{array}\right)=\left(\begin{array}{c}
\chi_{+} \\
0
\end{array}\right) \text {. }
$$

The spin operator along the $z$ direction is given by

$$
\Sigma^{3}=\sigma^{1^{2}}=\frac{i}{2}\left[\gamma^{1}, \gamma^{2}\right]=i \gamma^{1} \gamma^{2}=\left(\begin{array}{cc}
\sigma^{3} & 0 \\
0 & \sigma^{3}
\end{array}\right),
$$

where $\sigma$ with single index denotes Pauli spin matrices and that with double indices denotes the generator of the Lorentz group in spinor representation. Now,

$$
\begin{aligned}
\Sigma^{3} U_{R^{( \pm)}} & =\left(\begin{array}{cc}
\sigma^{3} & 0 \\
0 & \sigma^{3}
\end{array}\right)\left(\begin{array}{c}
0 \\
\chi_{ \pm}
\end{array}\right)=\left(\begin{array}{c}
0 \\
\sigma^{3} \chi_{ \pm}
\end{array}\right) \\
& = \pm\left(\begin{array}{c}
0 \\
\chi_{ \pm}
\end{array}\right)= \pm U_{R^{( \pm)}}, \\
\Sigma^{3} U_{L^{( \pm)}} & =\left(\begin{array}{cc}
\sigma^{3} & 0 \\
0 & \sigma^{3}
\end{array}\right)\left(\begin{array}{c}
\chi_{\mp} \\
0
\end{array}\right)=\left(\begin{array}{c}
\sigma^{3} \chi_{\mp} \\
0
\end{array}\right) \\
& =\mp\left(\begin{array}{c}
\chi_{\mp} \\
0
\end{array}\right)=\mp U_{L^{( \pm)}} .
\end{aligned}
$$

So, the modes $L^{(-)}$and $R^{(+)}$have spins along the direction of magnetic field whereas $L^{(+)}$and $R^{(-)}$have spins opposite to the direction of magnetic field. Now we discuss the helicity eigenstates of the various modes in LLL. The helicity operator is defined as

$$
\mathcal{H}_{\vec{p}}=\hat{\mathbf{p}} \cdot \vec{\Sigma}
$$

When a particle moves along $+z$ direction, $\hat{\mathbf{p}}=\hat{\mathbf{z}}$ and when it moves along $-z$ direction, $\hat{\mathbf{p}}=-\hat{\mathbf{z}}$. Thus,

$$
\mathcal{H}_{\vec{p}}= \begin{cases}\Sigma^{3}, & \text { for } p_{z}>0, \\ -\Sigma^{3}, & \text { for } p_{z}<0 .\end{cases}
$$

Thus,

$$
\mathcal{H}_{\vec{p}} U_{R^{( \pm)}}= \begin{cases} \pm U_{R^{( \pm)}}, & \text {for } p_{z}>0, \\ \mp U_{R^{( \pm)}}, & \text {for } p_{z}<0 .\end{cases}
$$

and

$$
\mathcal{H}_{\vec{p}} U_{L^{( \pm)}}= \begin{cases}\mp U_{L^{( \pm)},} & \text {for } p_{z}>0, \\ \pm U_{L^{( \pm)}}, & \text {for } p_{z}<0 .\end{cases}
$$




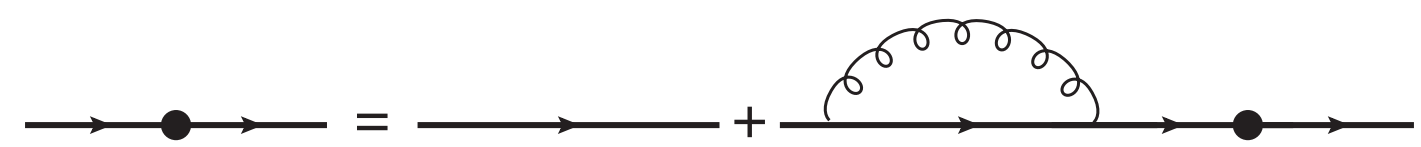

FIG. 1. Diagrammatic representation of the Dyson-Schwinger equation for one-loop effective fermion propagator.

\section{E. Dispersion}

In the presence of a magnetic field, the component of momentum transverse to the magnetic field is Landau quantized and takes discrete values given by $p_{\perp}^{2}=2 l\left|q_{f} B\right|$, where $l$ is a given Landau levels. In the presence of a pure background magnetic field and no heat bath $(T=0)$, the Dirac equation gives rise to a dispersion relation,

$$
E^{2}=p_{z}^{2}+m_{f}^{2}+(2 \nu+1) q_{f}|Q| B-q_{f} Q B \sigma,
$$

where $\nu=0,1,2, \ldots, Q= \pm 1, \sigma=+1$ for spin up and $\sigma=-1$ for spin down. The solutions are classified by energy eigenvalues,

$$
E_{l}^{2}=p_{z}^{2}+m_{f}^{2}+2 l q_{f} B,
$$

where one can define

$$
2 l=(2 \nu+1)|Q|-Q \sigma .
$$

Now we discuss the dispersion properties of a fermions in a hot magnetized medium. For the general case (for higher LLs, $l \neq 0$ ), the dispersion curves are obtained by solving $L^{2}=0$ and $R^{2}=0$ given in (30a) and (30b), numerically. We note that the roots of $L_{0}= \pm|\vec{L}| \Rightarrow L_{0} \mp|\vec{L}|=0$ are represented by $L^{( \pm)}$with energy $\omega_{L^{( \pm)}}$, whereas those for $R_{0}= \pm|\vec{R}| \Rightarrow R_{0} \mp|\vec{R}|=0$ by $R^{( \pm)}$with energy $\omega_{R^{( \pm)}}$. The corresponding eigenstates are obtained in (54a), (54b), (52a) and (52b) in subsection III D 1. We have chosen $T=0.2 \mathrm{GeV}, \alpha_{s}=0.3$ and $q_{f} B=0.5 m_{\pi}^{2}$, where $m_{\pi}$ is the pion mass. In Fig. 2, the dispersion curves for higher Landau levels are shown where all four modes can propagate for a given choice of $Q$. This is because the corresponding states for these modes are neither spin nor helicity eigenstates as shown in subsection III D 1. We also note that there will be negative energy modes which are not displayed here but would be discussed in the analysis of the spectral representation of the effective propagator Sec. V.

At LLL $l=0 \rightarrow p_{\perp}=0$ and the roots of $R_{0}= \pm R_{z}$ give rise to two right-handed modes $R^{( \pm)}$with energy $\omega_{R^{( \pm)}}$ whereas those for $L_{0}= \pm L_{z}$ produce ${ }^{4}$ two left-handed modes $L^{( \pm)}$with energy $\omega_{L^{( \pm)}}$. In Appendix D, the analytic solutions for the dispersion relations in LLL are presented which show four different modes and the corresponding

\footnotetext{
${ }^{4}$ We make a general note here for left-handed modes at LLL. At small $p_{z}, L_{z}$ itself is negative for LLL and becomes positive after a moderate value of $p_{z}$. This makes the left-handed modes $L^{(+)}$and $L^{(-)}$to flip in LLL than those in higher Landau levels. For details, see Appendix D.
}

eigenstates are obtained in subsection III D 2. Now, at LLL, we discuss two possibilities below:

(i) for positively charged fermion $Q=1, \sigma=1$ implies $\nu=0$ and $\sigma=-1$ implies $\nu=-1$. Now we note that $\nu$ can never be negative. This implies that the modes with $Q=1$ and $\sigma=-1$ (spin down) cannot propagate in LLL. Now, the right-handed mode $R^{(+)}$ and the left-handed mode $L^{(-)}$have spin up as shown in subsection III D 2, will propagate in LLL for $p_{z}>0$. The $R^{(+)}$mode has helicity to chirality ratio +1 is a quasiparticle whereas the mode $L^{(-)}$ left-handed has that of -1 known as plasmino (hole). However, for $p_{z}<0$, the right-handed mode flips to plasmino (hole) as its chirality to helicity ratio becomes -1 whereas the left-handed mode becomes particle as its chirality to helicity ratio becomes +1 . The dispersion behavior of the two modes are shown in the left panel of Fig. 3 which begins at mass $\left.m_{L L L}^{*-}\right|_{p_{z}=0}$ as given in (D13).

(ii) for negatively charged fermion $Q=-1, \sigma=1$ implies $\nu=-1$ and $\sigma=-1$ implies $\nu=0$. Thus, the modes with $Q=-1$ and $\sigma=+1$ (spin up) cannot propagate in LLL. However, the modes $L^{(+)}$and $R^{(-)}$have spin down as found in subsection IIID2 will propagate in LLL. Their dispersion are shown in the right panel of Fig. 3 which begin at mass $m_{L L L}^{*+}$ as given in (D13). For $p_{z}>0$, the mode $L^{(+)}$has helicity to chirality ratio +1 whereas $R^{(-)}$has that of -1 and vice-versa for $p_{z}<0$.

In the absence of the background magnetic field $(B=0)$, the two modes, the left-handed $L^{(+)}$and the right-handed $R^{(+)}$fermions, merge together whereas the other two modes, the left-handed $L^{(-)}$and the right-handed $R^{(-)}$ fermions, also merge together. This leads to degenerate (chirally symmetric) modes for which the dispersion plots start at $m_{t h}$ and one gets back the usual HTL result [49] with quasiparticle and plasmino modes in the presence of a heat bath as shown in Fig. 4.

As evident from the dispersion plots (Figs. 2 and 3 ) both left- and right-handed modes are also degenerate at $p_{z}=0$ in the presence of a magnetic field but at nonzero $\left|p_{z}\right|$ both left- and right-handed modes get separated from each others, causing a chiral asymmetry without disturbing the chiral invariance (subsection III C 1) in the system. Also in subsection III C 2 it was shown that the fermion propagator does not obey the reflection symmetry in the presence of a medium, which is now clearly evident from all dispersion plots as displayed above. 

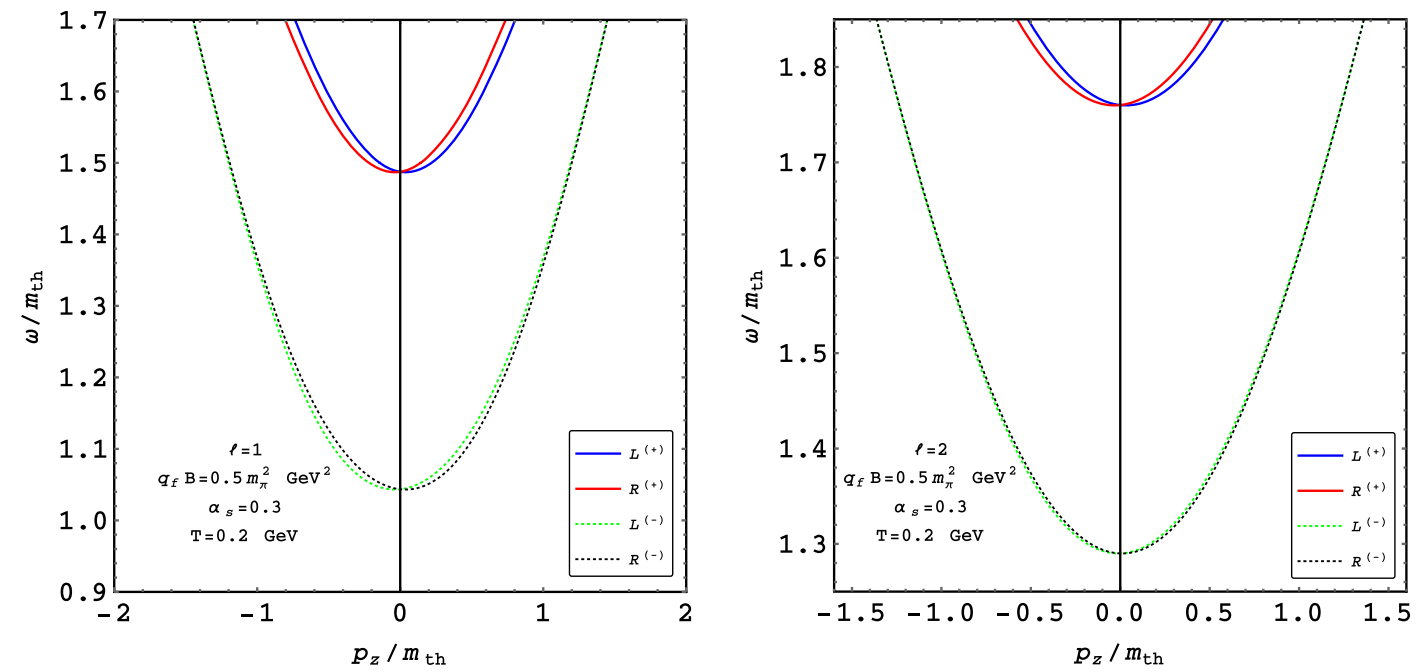

FIG. 2. Dispersion plots for higher Landau level, $l \neq 0$. The energy $\omega$ is scaled with the thermal mass $m_{t h}$ for convenience.
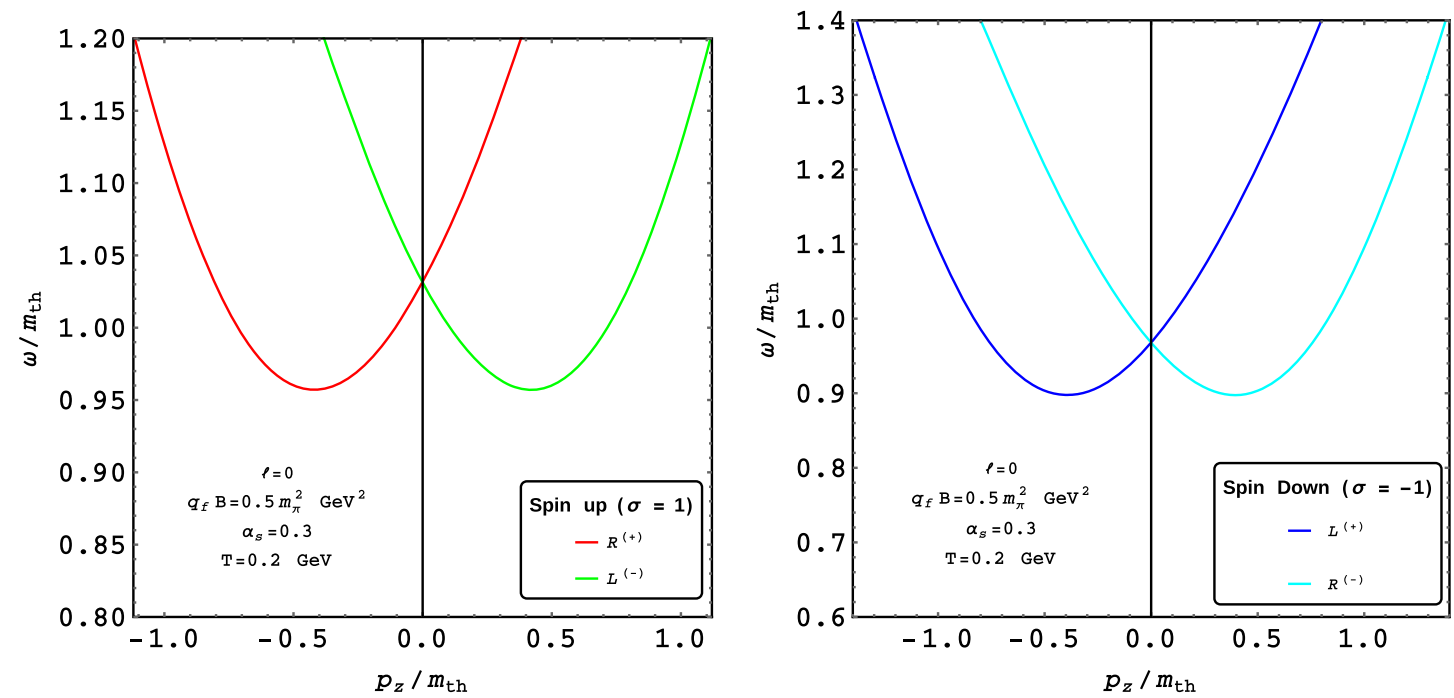

FIG. 3. Dispersion plots for LLL, $l=0$. The energy $\omega$ is scaled with the thermal mass $m_{t h}$ for convenience. For details, see the text.

\section{THREE-POINT FUNCTION}

The $(N+1)$-point functions are related to the $N$-point functions through Ward-Takahashi (WT) identity. The three-point function is related to the two-point function as

$$
\begin{aligned}
& Q_{\mu} \Gamma^{\mu}(P, K ; Q)=S^{-1}(P)-S^{-1}(K)=P P-\not K-\Sigma(P)+\Sigma(K) \\
& =\underbrace{(\not P-\not K)}_{\text {Free }}-\underbrace{\left(\Sigma^{B=0}(P, T)-\Sigma^{B=0}(K, T)\right)}_{\text {Thermal or HTL correction }}-\underbrace{\left(\Sigma^{B \neq 0}(P, T)-\Sigma^{B \neq 0}(K, T)\right)}_{\text {Thermomagnetic correction }} \\
& =\mathscr{Q}+a\left(p_{0},|\vec{p}|\right) \not P+b\left(p_{0},|\vec{p}|\right) \not \iota-a\left(k_{0},|\vec{k}|\right) K-b\left(k_{0},|\vec{k}|\right) \not \iota+b^{\prime}\left(p_{0}, p_{\perp}, p_{z}\right) \gamma_{5} \not h \\
& +c^{\prime}\left(p_{0}, p_{\perp}, p_{z}\right) \gamma_{5} \not h-b^{\prime}\left(k_{0}, k_{\perp}, k_{z}\right) \gamma_{5} \not h-c^{\prime}\left(k_{0}, k_{\perp}, k_{z}\right) \gamma_{5} \not h,
\end{aligned}
$$


where $Q=P-K$. We note that recently the general form of the thermomagnetic corrections for three-point $[66,67]$ and four-point [67] functions have been given in terms of the involved angular integrals, which satisfy WT identities. Nevertheless, to validate the general structure of the self-energy in (16) vis-a-vis the inverse propagator in (25), we obtain below the temporal component of the three-point function at $\vec{q}=0 ; \vec{p}=\vec{k}$ and $p=k$.

Using (23a), (23b), (23c) and (23d), we can obtain

$$
\begin{aligned}
\left.\Gamma^{0}(P, K ; Q)\right|_{\vec{q}=0} & =\gamma_{0}-\underbrace{-\frac{m_{t h}^{2}}{p q_{0}} \delta Q_{0} \gamma^{0}+\frac{m_{t h}^{2}}{p q_{0}} \delta Q_{1}(\hat{p} \cdot \vec{\gamma})}_{\text {Thermal or HTL correction }} \underbrace{-\frac{M^{\prime 2}}{p q_{0}}\left[\delta Q_{0} \gamma_{5}+\frac{p_{z}}{p} \delta Q_{1}\left(i \gamma^{1} \gamma^{2}\right)\right] \gamma^{3}}_{\text {Thermomagnetic correction }} \\
& =\gamma^{0}+\delta \Gamma_{\mathrm{HTL}}^{0}(P, K ; Q)+\delta \Gamma_{\mathrm{TM}}^{0}(P, K ; Q),
\end{aligned}
$$

with

$$
\begin{aligned}
\gamma_{5} \gamma^{0} & =-i \gamma^{1} \gamma^{2} \gamma^{3}, \\
M^{\prime 2} & =4 C_{F} g^{2} M^{2}\left(T, m, q_{f} B\right), \\
\delta Q_{j} & =Q_{j}\left(\frac{p_{0}}{p}\right)-Q_{j}\left(\frac{k_{0}}{p}\right) .
\end{aligned}
$$

where $Q_{j}$ are the Legendre functions of the second kind given in (A7a) and (A7b). Important to note that the thermomagnetic (TM) correction $\delta \Gamma_{\mathrm{TM}}^{0}$ matches exactly with that from direct calculation in (C5) in Appendix C. The result also agrees with the HTL three-point function $[66,67]$ in the absence of the background magnetic field by setting $B=0 \Rightarrow M^{\prime}=0$ as

$$
\begin{aligned}
\left.\Gamma_{\mathrm{HTL}}^{0}(P, K ; Q)\right|_{\vec{q}=0} & =\left[1-\frac{m_{t h}^{2}}{p q_{0}} \delta Q_{0}\right] \gamma^{0}+\frac{m_{t h}^{2}}{p q_{0}} \delta Q_{1}(\hat{p} \cdot \vec{\gamma}) \\
& =\gamma^{0}+\delta \Gamma_{\mathrm{HTL}}^{0}(P, K ; Q),
\end{aligned}
$$

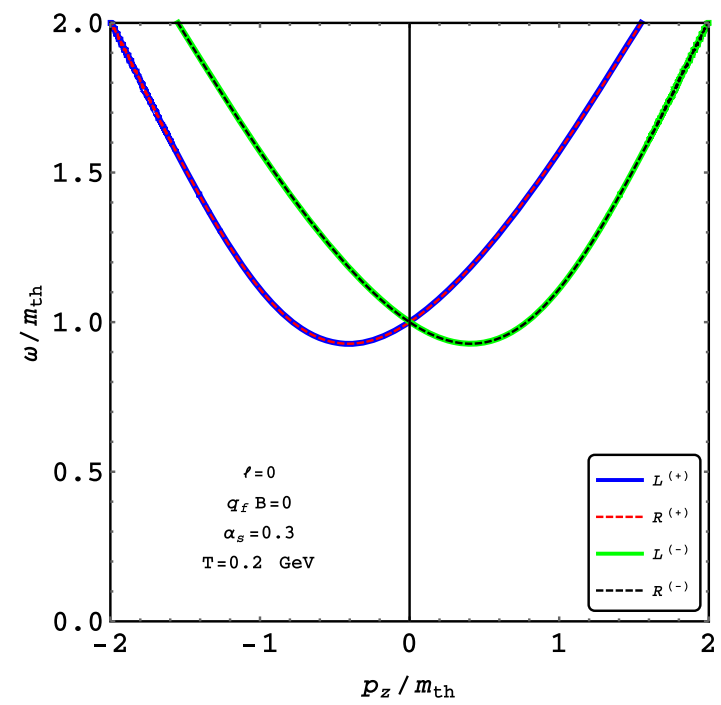

FIG. 4. The dispersion plots corresponding to HTL propagator in the absence of a magnetic field, i.e., $B=0$. where all components, i.e., $(0,1,2,3)$, are relevant for a pure thermal background.

Now in the absence of a heat bath, setting $T=0 \Rightarrow$ $m_{t h}=0$ and $M^{\prime 2}=4 C_{F} g^{2} M^{2}\left(T=0, m, q_{f}, B\right)$, the temporal three-point function in (68) reduces to

$$
\begin{aligned}
\left.\Gamma_{B}^{0}(P, K ; Q)\right|_{\vec{q}=0} & =\gamma^{0} \underbrace{-\frac{M^{\prime 2}}{p q_{0}}\left[\delta Q_{0} \gamma_{5}+\frac{p p_{z}}{p} \delta Q_{1}\left(i \gamma^{1} \gamma^{2}\right)\right] \gamma^{3}}_{\text {Pure magnetic correction }} \\
& =\gamma^{0}+\delta \Gamma_{\mathrm{M}}^{0}(P, K ; Q) .
\end{aligned}
$$

We now note that this is the three-point function with pure background magnetic field but no heat bath. The gauge boson is oriented along the field direction and there is no polarization in the transverse direction. Thus, only the longitudinal components (i.e., $(0,3)$ components) of the three-point function would be relevant for pure background magnetic field in contrast to that of (70) for pure thermal background.

\section{SPECTRAL REPRESENTATION OF THE EFFECTIVE PROPAGATOR}

In this section, we obtain the spectral representation of the effective propagator in a hot magnetized medium. This quantity is of immense interest for studying the various spectral properties, real and virtual photon production, damping rates and various transport coefficients etc. of the hot magnetized medium, in particular, for hot magnetized QCD medium.

\section{A. General case}

The effective propagator as obtained in (29) is given by

$$
S^{*}=\mathcal{P}_{-} \frac{\not L}{L^{2}} \mathcal{P}_{+}+\mathcal{P}_{+} \frac{\not R}{R^{2}} \mathcal{P}_{-},
$$

where $\not K$ and $\not R$ can be written in the rest frame of the heat bath and the magnetic field in the $z$-direction following (27a) and (27b), respectively, as 


$$
\begin{aligned}
\not L= & {\left[\left(1+a\left(p_{0}, p\right)\right) p_{0}+b\left(p_{0}, p\right)+b^{\prime}\left(p_{0}, p_{\perp}, p_{z}\right)\right] \gamma^{0}-\left[\left(1+a\left(p_{0}, p\right)\right) p_{z}+c^{\prime}\left(p_{0}, p_{\perp}, p_{z}\right)\right] \gamma^{3} } \\
& -\left(1+a\left(p_{0}, p\right)\right)(\gamma \cdot p)_{\perp} \\
= & {\left[\left(1+a\left(p_{0}, p\right)\right) p_{0}+b\left(p_{0}, p\right)+b^{\prime}\left(p_{0}, p_{\perp}, p_{z}\right)\right] \gamma^{0}-\left[p\left(1+a\left(p_{0}, p\right)\right)\right](\gamma \cdot \hat{p})-c^{\prime}\left(\left(p_{0}, p_{\perp}, p_{z}\right) \gamma^{3}\right.} \\
= & g_{L}^{1}\left(p_{0}, p_{\perp}, p_{z}\right) \gamma^{0}-g_{L}^{2}\left(p_{0}, p_{\perp}, p_{z}\right)(\gamma \cdot \hat{p})-g_{L}^{3}\left(p_{0}, p_{\perp}, p_{z}\right) \gamma^{3}, \\
\not R= & {\left[\left(1+a\left(p_{0}, p\right)\right) p_{0}+b\left(p_{0}, p\right)-b^{\prime}\left(p_{0}, p_{\perp}, p_{z}\right)\right] \gamma^{0}-\left[\left(1+a\left(p_{0}, p\right)\right) p_{z}-c^{\prime}\left(p_{0}, p_{\perp}, p_{z}\right)\right] \gamma^{3} } \\
& \times\left(-1+a\left(p_{0}, p\right)\right)(\gamma \cdot p)_{\perp} \\
= & {\left[\left(1+a\left(p_{0}, p\right)\right) p_{0}+b\left(p_{0}, p\right)-b^{\prime}\left(p_{0}, p_{\perp}, p_{z}\right)\right] \gamma^{0}-\left[p\left(1+a\left(p_{0}, p\right)\right)\right](\gamma \cdot \hat{p})+c^{\prime}\left(p_{0}, p_{\perp}, p_{z}\right) \gamma^{3} } \\
= & g_{R}^{1}\left(p_{0}, p_{\perp}, p_{z}\right) \gamma^{0}-g_{R}^{2}\left(p_{0}, p_{\perp}, p_{z}\right)(\gamma \cdot \hat{p})+g_{R}^{3}\left(p_{0}, p_{\perp}, p_{z}\right) \gamma^{3},
\end{aligned}
$$

where $\hat{p}=\mathbf{p} /|\mathbf{p}|, p=|\mathbf{p}|$ and, $p_{z}$ and $p_{\perp}$ are given, respectively, in (22b) and (22c). We also note that though $g_{L}^{2}=g_{R}^{2}$; $g_{L}^{3}=g_{R}^{3}$, but they are treated separately for the sake of notations that we would be using, for convenience, as $g_{L}^{i}$ and $g_{R}^{i}$. One can decompose the effective propagator into six parts by separating out the $\gamma$ matrices as

$$
\begin{aligned}
S^{*}= & \mathcal{P}_{-} \gamma^{0} \mathcal{P}_{+} \frac{g_{L}^{1}\left(p_{0}, p_{\perp}, p_{z}\right)}{L^{2}}-\mathcal{P}_{-}(\gamma \cdot \hat{p}) \mathcal{P}_{+} \frac{g_{L}^{2}\left(p_{0}, p_{\perp}, p_{z}\right)}{L^{2}}-\mathcal{P}_{-} \gamma^{3} \mathcal{P}_{+} \frac{g_{L}^{3}\left(p_{0}, p_{\perp}, p_{z}\right)}{L^{2}} \\
& +\mathcal{P}_{+} \gamma^{0} \mathcal{P}_{-} \frac{g_{R}^{1}\left(p_{0}, p_{\perp}, p_{z}\right)}{R^{2}}-\mathcal{P}_{+}(\gamma \cdot \hat{p}) \mathcal{P}_{-} \frac{g_{R}^{2}\left(p_{0}, p_{\perp}, p_{z}\right)}{R^{2}}+\mathcal{P}_{+} \gamma^{3} \mathcal{P}_{-} \frac{g_{R}^{3}\left(p_{0}, p_{\perp}, p_{z}\right)}{R^{2}}
\end{aligned}
$$

In subsection III E, we have discussed that $L^{2}=0$ yields four poles, leading to four modes with both positive and negative energy as $\pm \omega_{L^{(+)}}\left(p_{\perp}, p_{z}\right)$ and $\pm \omega_{L^{(-)}}\left(p_{\perp}, p_{z}\right)$. Similarly, $R^{2}=0$ also yields four poles, namely $\pm \omega_{R^{(+)}}\left(p_{\perp}, p_{z}\right)$ and $\pm \omega_{R^{(-)}}\left(p_{\perp}, p_{z}\right)$.

With this information one can obtain the spectral representation $[49,72-74]$ of the effective propagator in (76) as

$$
\rho=\left(\mathcal{P}_{-} \gamma^{0} \mathcal{P}_{+}\right) \rho_{L}^{1}-\left(\mathcal{P}_{-}(\gamma \cdot \hat{p}) \mathcal{P}_{+}\right) \rho_{L}^{2}-\left(\mathcal{P}_{-} \gamma^{3} \mathcal{P}_{+}\right) \rho_{L}^{3}+\left(\mathcal{P}_{+} \gamma^{0} \mathcal{P}_{-}\right) \rho_{R}^{1}-\left(\mathcal{P}_{+}(\gamma \cdot \hat{p}) \mathcal{P}_{-}\right) \rho_{R}^{2}+\left(\mathcal{P}_{+} \gamma^{3} \mathcal{P}_{-}\right) \rho_{R}^{3}
$$

where the spectral function corresponding to each of the term can be written as

$$
\begin{aligned}
\rho_{L}^{i}= & \frac{1}{\pi} \operatorname{Im}\left(\frac{g_{L}^{i}}{L^{2}}\right) \\
= & Z_{L^{(+)}}^{i+}\left(p_{\perp}, p_{z}\right) \delta\left(p_{0}-\omega_{L^{(+)}}\left(p_{\perp}, p_{z}\right)\right)+Z_{L^{(+)}}^{i-}\left(p_{\perp}, p_{z}\right) \delta\left(p_{0}+\omega_{L^{(+)}}\left(p_{\perp}, p_{z}\right)\right) \\
& +Z_{L^{(-)}}^{i+}\left(p_{\perp}, p_{z}\right) \delta\left(p_{0}-\omega_{L^{(-)}}\left(p_{\perp}, p_{z}\right)\right)+Z_{L^{(-)}}^{i-}\left(p_{\perp}, p_{z}\right) \delta\left(p_{0}+\omega_{L^{(-)}}\left(p_{\perp}, p_{z}\right)\right)+\beta_{L}^{i}, \\
\rho_{R}^{i}= & \frac{1}{\pi} \operatorname{Im}\left(\frac{g_{R}^{i}}{R^{2}}\right) \\
= & Z_{R^{(+)}}^{i+}\left(p_{\perp}, p_{z}\right) \delta\left(p_{0}-\omega_{R^{(+)}}\left(p_{\perp}, p_{z}\right)\right)+Z_{R^{(+)}}^{i-}\left(p_{\perp}, p_{z}\right) \delta\left(p_{0}+\omega_{R^{(+)}}\left(p_{\perp}, p_{z}\right)\right) \\
& +Z_{R^{(-)}}^{i+}\left(p_{\perp}, p_{z}\right) \delta\left(p_{0}-\omega_{R^{(-)}}\left(p_{\perp}, p_{z}\right)\right)+Z_{R^{(-)}}^{i-}\left(p_{\perp}, p_{z}\right) \delta\left(p_{0}+\omega_{R^{(-)}}\left(p_{\perp}, p_{z}\right)\right)+\beta_{R}^{i},
\end{aligned}
$$

where $i=1,2,3$. We note that the delta functions are associated with pole parts originating from the timelike domain $\left(p_{0}^{2}>p^{2}\right)$, whereas the cut parts $\beta_{L(R)}^{i}$ are associated with the Landau damping arises from the spacelike domain, $p_{0}^{2}<p^{2}$, of the propagator. The residues $Z_{L(R)}^{i}$ are determined at the various poles as

$$
Z_{L(R)}^{i \text { sgn of pole }}\left(p_{\perp}, p_{z}\right)=g_{L(R)}^{i}\left(p_{0}, p\right)\left|\frac{\partial L^{2}\left(R^{2}\right)}{\partial p_{0}}\right|_{p_{0}=\text { pole }}^{-1}
$$

As a demonstration, we present analytical expressions of three residues corresponding to the pole $p_{0}=+\omega_{L^{(+)}}$as 

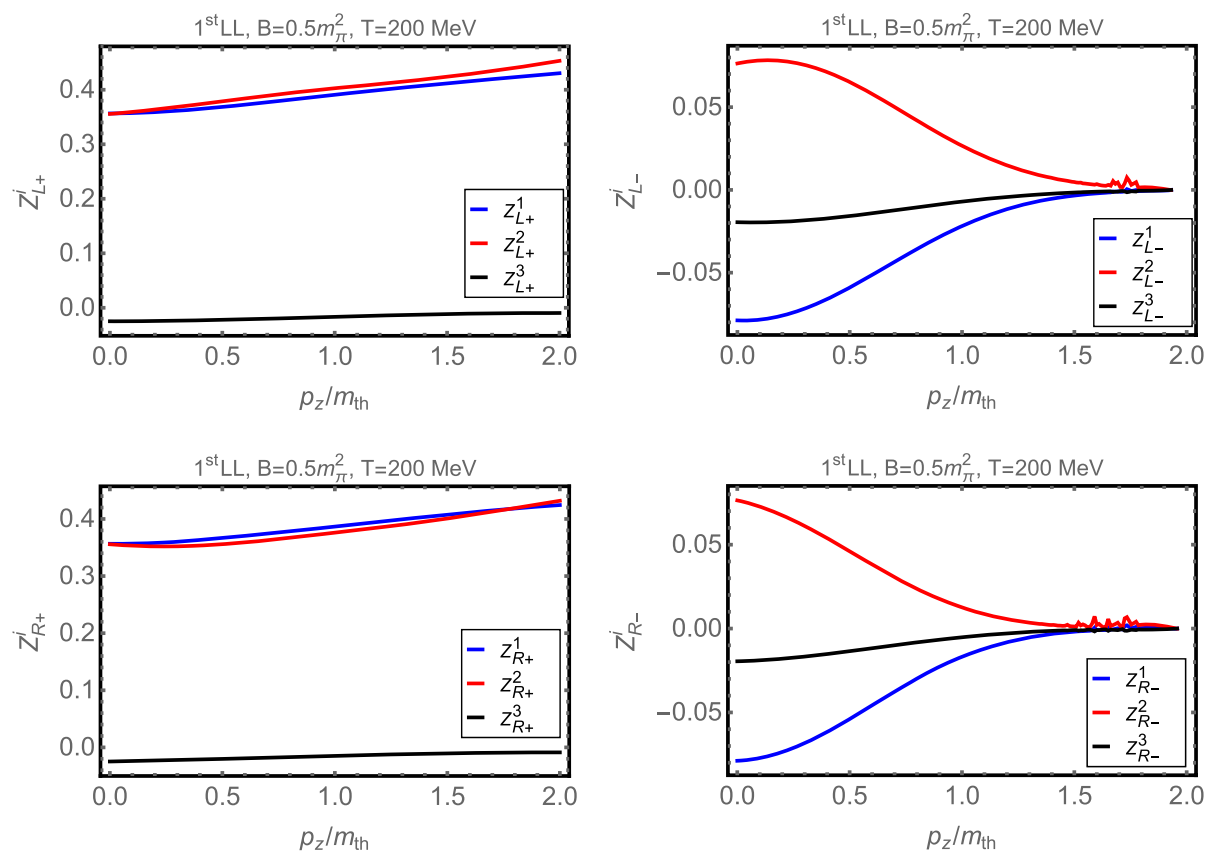

FIG. 5. Different Residues for the first LL $(l=1)$ are plotted with scaled momentum along the magnetic field direction.

$$
\begin{aligned}
Z_{L^{(+)}}^{1+} & =\frac{p\left(p^{2}-\omega_{L^{(+)}}^{2}\right)\left[p^{2}\left(m_{t h}^{2} \log \left(\frac{\omega_{L^{(+)}}+p}{\omega_{L^{(+)}}-p}\right)-2 p \omega_{L^{(+)}}\right)+M^{\prime 2} p_{z}\left(2 p-\omega_{L^{(+)}}\right) \log \left(\frac{\omega_{L^{(+)}}+p}{\omega_{L^{(+)}}-p}\right)\right]}{m_{t h}^{2}\left[8 p^{4}\left(\omega_{L^{(+)}}+M^{\prime 2} / m_{t h}^{2} p_{z}\right)+\log \left(\frac{\omega_{L^{(+)}}+p}{\omega_{L^{(+)}}-p}\right) X\right]}, \\
Z_{L^{(+)}}^{2+} & =\frac{p^{2}\left(p^{2}-\omega_{L^{(+)}}^{2}\right)\left[2 p\left(m_{t h}^{2}+p^{2}\right)-m_{t h}^{2} \omega_{L^{(+)}} \log \left(\frac{\omega_{L^{(+)}}+p}{\omega_{L^{(+)}}-p}\right)\right]}{m_{t h}^{2}\left[8 p^{4}\left(\omega_{L^{(+)}}+M^{\prime 2} / m_{t h}^{2} p_{z}\right)+\log \left(\frac{\omega_{L^{(+)}}+p}{\omega_{L^{(+)}}-p}\right) X\right]} \\
Z_{L^{(+)}}^{3+} & =\frac{-M^{\prime 2} p^{5}\left(p^{2}-\omega_{L^{(+)}}^{2}\right) \log \left(\frac{\omega_{L^{(+)}}+p}{\omega_{L^{(+)}}-p}\right)}{m_{t h}^{4}\left[8 p^{4}\left(\omega_{L^{(+)}}+M^{\prime 2} / m_{t h}^{2} p_{z}\right)+\log \left(\frac{\omega_{L^{(+)}}+p}{\omega_{L^{(+)}}-p}\right) X\right]}
\end{aligned}
$$

where $X=2 p^{3}\left(M^{\prime 2}-2 m_{t h}^{2}\right)+2 M^{\prime 2} p p_{z}^{2}+M^{\prime 2} \omega_{L^{(+)}} p_{\perp}^{2} \log \left(\frac{\omega_{L^{(+)}}+p}{\omega_{L^{(+)}}-p}\right)$. The other poles of $L^{2}=0$ can trivially be found out by replacing $\omega_{L^{(+)}}$in the above expressions. The expressions for the residues for $R$ parts can similarly be expressed as the $L$ parts, but we do not show them.

Below, in Fig. 5, we present the residues corresponding to the first Landau level where all the terms are present. We take the value of the magnetic field as $m_{\pi}^{2} / 2$ and temperature to be $200 \mathrm{MeV}$.

Now, the expressions for the cut parts $\beta_{L(R)}^{i}$ are given below:

$\beta_{L}^{i}=\frac{1}{\pi} \Theta\left(p^{2}-p_{0}^{2}\right) \frac{\operatorname{Im}\left(g_{L}^{i}\right) \operatorname{Re}\left(L^{2}\right)-\operatorname{Im}\left(L^{2}\right) \operatorname{Re}\left(g_{L}^{i}\right)}{\left(\operatorname{Re}\left(L^{2}\right)\right)^{2}+\left(\operatorname{Im}\left(L^{2}\right)\right)^{2}}$,

$\beta_{R}^{i}=\frac{1}{\pi} \Theta\left(p^{2}-p_{0}^{2}\right) \frac{\operatorname{Im}\left(g_{R}^{i}\right) \operatorname{Re}\left(R^{2}\right)-\operatorname{Im}\left(R^{2}\right) \operatorname{Re}\left(g_{R}^{i}\right)}{\left(\operatorname{Re}\left(R^{2}\right)\right)^{2}+\left(\operatorname{Im}\left(R^{2}\right)\right)^{2}}$,

where

$$
\operatorname{Re}\left(g_{L}^{1}\right)=p_{0}-M^{\prime 2} \frac{p_{z}}{p^{2}}-\frac{m_{t h}^{2}}{p}\left(1-\frac{M^{\prime 2}}{m_{t h}^{2}} \frac{p_{z} p_{0}}{p^{2}}\right) Q_{0}\left(\left|\frac{p_{0}}{p}\right|\right),
$$

$\operatorname{Im}\left(g_{L}^{1}\right)=\frac{\pi}{2} \frac{m_{\text {th }}^{2}}{p}\left(1-\frac{M^{\prime 2}}{m_{t h}^{2}} \frac{p_{z} p_{0}}{p^{2}}\right)$,

$\operatorname{Re}\left(g_{L}^{2}\right)=\operatorname{Re}\left(g_{R}^{2}\right)=p+\frac{m_{\text {th }}^{2}}{p}\left[1-\frac{p_{0}}{p} Q_{0}\left(\left|\frac{p_{0}}{p}\right|\right)\right]$,

$\operatorname{Im}\left(g_{L}^{2}\right)=\operatorname{Im}\left(g_{R}^{2}\right)=\pi m_{t h}^{2} \frac{p_{0}}{2 p^{2}}$,

$\operatorname{Re}\left(g_{L}^{3}\right)=\operatorname{Re}\left(g_{R}^{3}\right)=\frac{M^{\prime 2}}{p} Q_{0}\left(\left|\frac{p_{0}}{p}\right|\right)$, 
$\operatorname{Im}\left(g_{L}^{3}\right)=\operatorname{Im}\left(g_{R}^{3}\right)=-\frac{\pi M^{\prime 2}}{2 p}$,

and

$\operatorname{Re}\left(g_{R}^{1}\right)=p_{0}+M^{\prime 2} \frac{p_{z}}{p^{2}}-\frac{m_{t h}^{2}}{p}\left(1+\frac{M^{\prime 2}}{m_{t h}^{2}} \frac{p_{z} p_{0}}{p^{2}}\right) Q_{0}\left(\left|\frac{p_{0}}{p}\right|\right)$,

$\operatorname{Im}\left(g_{R}^{1}\right)=\frac{\pi}{2} \frac{m_{t h}^{2}}{p}\left(1+\frac{M^{\prime 2}}{m_{t h}^{2}} \frac{p_{z} p_{0}}{p^{2}}\right)$.

Also, we obtain

$\operatorname{Re}\left(L^{2}\right)=A_{L}+B_{L} Q_{0}\left(\left|\frac{p_{0}}{p}\right|\right)+C\left(Q_{0}^{2}\left(\left|\frac{p_{0}}{p}\right|\right)-\frac{\pi^{2}}{4}\right)$,

$\operatorname{Im}\left(L^{2}\right)=-\frac{\pi B_{L}}{2}-\pi Q_{0}\left(\left|\frac{p_{0}}{p}\right|\right) C$,

$\operatorname{Re}\left(R^{2}\right)=A_{R}+B_{R} Q_{0}\left(\left|\frac{p_{0}}{p}\right|\right)+C\left(Q_{0}^{2}\left(\left|\frac{p_{0}}{p}\right|\right)-\frac{\pi^{2}}{4}\right)$,

$\operatorname{Im}\left(R^{2}\right)=-\frac{\pi B_{R}}{2}-\pi Q_{0}\left(\left|\frac{p_{0}}{p}\right|\right) C$

where
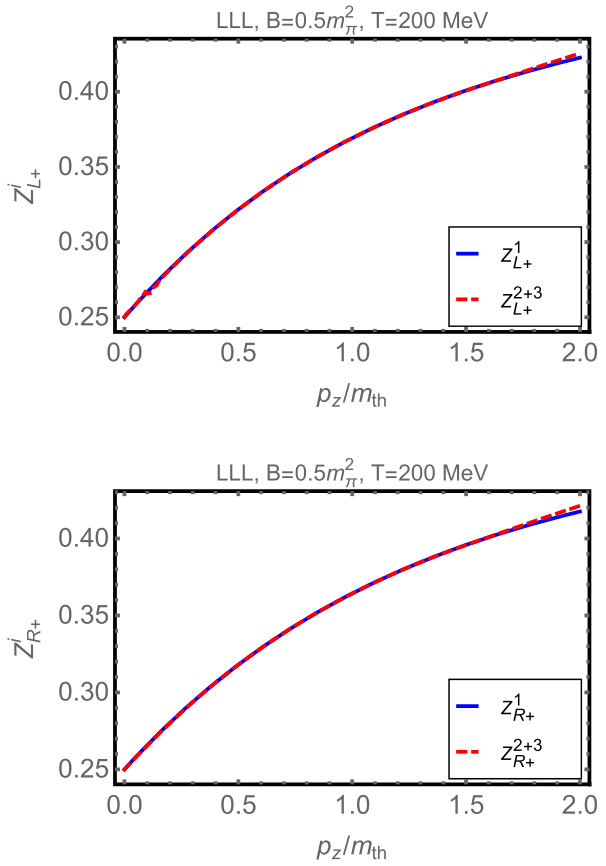

$A_{L}=p_{0}^{2}-p^{2}-2 m_{t h}^{2}-\frac{m_{t h}^{4}}{p^{2}}-\frac{2 M^{\prime 2} p_{0} p_{z}}{p^{2}}+\frac{M^{\prime 4} p_{z}^{2}}{p^{4}}$,

$A_{R}=p_{0}^{2}-p^{2}-2 m_{t h}^{2}-\frac{m_{t h}^{4}}{p^{2}}+\frac{2 M^{\prime 2} p_{0} p_{z}}{p^{2}}+\frac{M^{\prime 4} p_{z}^{2}}{p^{4}}$,

$B_{L}=\frac{2 m_{t h}^{4} p_{0}}{p^{3}}-\frac{2 M^{\prime 2} p_{z}}{p}+\frac{2 M^{\prime 2} p_{0}^{2} p_{z}}{p^{3}}-\frac{2 M^{\prime 4} p_{0} p_{z}^{2}}{p^{5}}$,

$B_{R}=\frac{2 m_{t h}^{4} p_{0}}{p^{3}}+\frac{2 M^{\prime 2} p_{z}}{p}-\frac{2 M^{\prime 2} p_{0}^{2} p_{z}}{p^{3}}-\frac{2 M^{\prime 4} p_{0} p_{z}^{2}}{p^{5}}$,

$C=\frac{m_{t h}^{4}-M^{\prime 4}}{p^{2}}-\frac{p_{0}^{2} m_{t h}^{4}}{p^{4}}+\frac{M^{\prime 4} p_{0}^{2} p_{z}^{2}}{p^{6}}$.

\section{B. LLL case}

For LLL, as $p_{\perp}=0$, so $g_{L(R)}^{2}$ and $g_{L(R)}^{3}$ in (74) and (75) can now be merged as

$$
\begin{aligned}
& g_{L}^{2+3}=\left[\left(1+a\left(p_{0}, p\right)\right) p_{z}+c^{\prime}\left(p_{0}, p\right)\right] \gamma^{3}, \\
& g_{R}^{2+3}=\left[\left(1+a\left(p_{0}, p\right)\right) p_{z}-c^{\prime}\left(p_{0}, p\right)\right] \gamma^{3} .
\end{aligned}
$$

The spectral function corresponding to LLL reads as

$$
\begin{aligned}
\rho_{\mathrm{LLL}}= & \left(\mathcal{P}_{-} \gamma^{0} \mathcal{P}_{+}\right) \rho_{L}^{1}-\left(\mathcal{P}_{-} \gamma^{3} \mathcal{P}_{+}\right) \rho_{L}^{2+3} \\
& +\left(\mathcal{P}_{+} \gamma^{0} \mathcal{P}_{-}\right) \rho_{R}^{1}-\left(\mathcal{P}_{+} \gamma^{3} \mathcal{P}_{-}\right) \rho_{R}^{2+3} .
\end{aligned}
$$

where one needs to determine
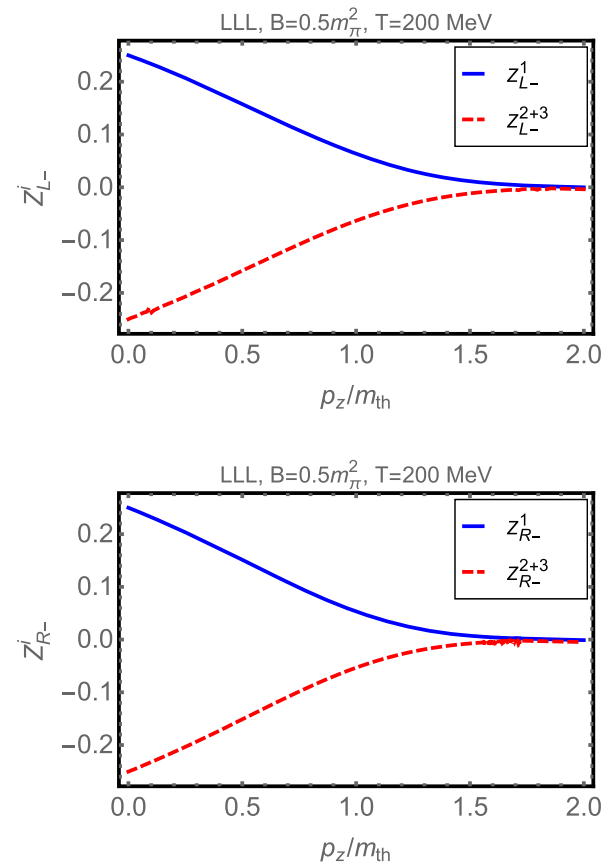

FIG. 6. Different Residues for the LLL $(l=0)$ are plotted with scaled momentum along the magnetic field direction. 


$$
\rho_{L(R)}^{2+3}=\frac{1}{\pi} \operatorname{Im}\left(\frac{g_{L(R)}^{2+3}}{L^{2}\left(R^{2}\right)}\right),
$$

which can again be represented in terms of different residues corresponding to different poles of $L^{2}\left(R^{2}\right)=0$ as in Eq. (79). In Fig. 6, the variation of the residues for the lowest Landau level are shown.

In Appendix E, we have demonstrated how one gets back the HTL spectral functions when magnetic field is withdrawn from the thermal medium.

\section{CONCLUSIONS}

In this article, the general structure of fermionic selfenergy for a chirally invariant theory has been formulated for a hot and magnetized medium. Using this we have obtained a closed form of the general structure of the effective fermion propagator. The collective excitations in such a nontrivial background have been obtained for timelike momenta in the weak-field and HTL approximation in the domain $m_{t h}^{2}\left(\sim g^{2} T^{2}<\left|q_{f} B\right|<T^{2}\right.$. We found that the left- and right-handed modes get separated and become asymmetric in the presence of a magnetic field which were degenerate and symmetric otherwise. The transformation of the effective propagator in a hot magnetized medium under some of the discrete symmetries have been studied and its consequences are also reflected in the collective fermion modes in the Landau levels. We have also obtained the Dirac spinors of the various collective modes by solving the Dirac equation with the effective twopoint function. Further, we checked the general structure of the two-point function by obtaining the three-point function using the Ward-Takahashi identity, which agrees with the direct calculation of one-loop order in weak-field approximation. We also found that only the longitudinal component of the vertex would be relevant when there is only background magnetic field. The spectral function corresponding to the effective propagator is explicitly obtained for a hot magnetized medium which will be extremely useful for studying the spectral properties, e.g., photon/ dilepton production, damping rate, and transport coefficients for a hot magnetized medium. This has pole contribution due to the various collective modes originating from the timelike domain and a Landau cut contribution appearing from the spacelike domain. It has explicitly been shown that the spectral function reduces to that obtained for thermal medium in the absence of the magnetic field. Our formulation is in general applicable to both QED and QCD with nontrivial background such as a hot magnetized medium.

\section{ACKNOWLEDGMENTS}

The authors would like to acknowledge useful discussions with Palash B Pal, Najmul Haque, Chowdhury Aminul Islam, Arghya Mukherjee and Bithika Karmakar.
A. B. and M. G. M. were funded by the Department of Atomic Energy (DAE), India, via the project TPAES, whereas A.D. and P.K.R. were funded by the project DAE/ALICE/SINP. A. B. was also partially supported by the National Post Doctoral Program CAPES (PNPD/ CAPES), Govt. of Brazil.

\section{APPENDIX A: COMPUTATIONS OF STRUCTURE FUNCTIONS IN ONE LOOP IN A WEAK-FIELD APPROXIMATION FOR A HOT MAGNETIZED QCD MEDIUM}

Here, we present the computations of the various structure functions in (21a) to (21d) in one-loop order (Fig. 7) in a weak-field and HTL approximations following the imaginary time formalism. In Fig. 7, the modified quark propagator (bold line) due to background magnetic field is given in (A3). Since glouns are chargeless, their propagators do not change in the presence of a magnetic field. The gluon propagator in Feynman gauge, is given as [41]

$$
D_{a b}^{\mu \nu}(Q)=-i \delta_{a b} \frac{g^{\mu \nu}}{Q^{2}}
$$

We note that we would like to explore the fermion spectrum in a hot magnetized background in the limit $m_{f}^{2}<q_{f} B<$ $T^{2}$. In this domain, the fermion propagator is obtained by expanding the sum over all Landau levels in powers of $q_{f} B$ in (7) and keeping up to $\mathcal{O}\left(\left[q_{f} B\right)^{2}\right]$, it reads as

$$
\begin{aligned}
S(K)= & i \frac{K+m_{f}}{K^{2}-m^{2}}+i \gamma_{1} \gamma_{2} \frac{K_{\|}+m_{f}}{\left(K^{2}-m_{f}^{2}\right)^{2}} q_{f} B \\
& +2\left[\frac{K_{\|}+m_{f}}{\left(K^{2}-m_{f}^{2}\right)^{4}} K_{\perp}^{2}-\frac{K_{\|}^{2}-m_{f}^{2}}{\left(K^{2}-m_{f}^{2}\right)^{4}} K_{\perp}\right]\left(q_{f} B\right)^{2} \\
= & i \frac{K+m_{f}}{K^{2}-m_{f}^{2}}+i \gamma_{1} \gamma_{2} \frac{K_{\|}+m_{f}}{\left(K^{2}-m_{f}^{2}\right)^{2}} q_{f} B+\mathcal{O}\left[\left(q_{f} B\right)^{2}\right],
\end{aligned}
$$

where the first term is the free propagator and the second one is $\mathcal{O}\left[q_{f} B\right]$ correction to it. Now combining (A2) and (11) the fermion propagator in background magnetic field reads as

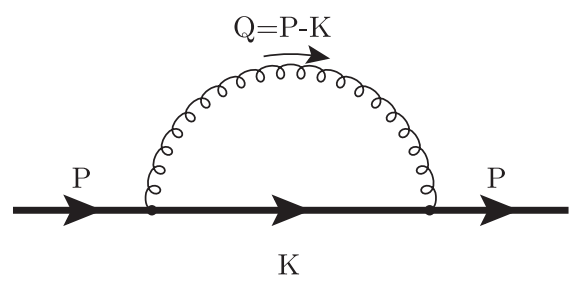

FIG. 7. One-loop fermion self-energy in a hot magnetized medium. 


$$
\begin{aligned}
S(K)= & i \frac{K}{K^{2}-m_{f}^{2}}-\frac{\gamma_{5}[(K . n) \not h-(K . u) \not h]}{\left(K^{2}-m_{f}^{2}\right)^{2}} \\
& \times\left(q_{f} B\right)+\mathcal{O}\left[\left(q_{f} B\right)^{2}\right] \\
= & S_{1}^{B=0}(K)+S_{2}^{B \neq 0}(K)+\mathcal{O}\left[\left(q_{f} B\right)^{2}\right],
\end{aligned}
$$

where the fermion mass in the numerator has been neglected in the weak-field domain, $m_{f}^{2}<\left(q_{f} B\right)<T^{2}$.

The one-loop quark self-energy up to $\mathcal{O}\left(\left|q_{f} B\right|\right)$ can be written as

$$
\begin{aligned}
\Sigma(P)= & g^{2} C_{F} T \mathcal{Y}_{\{K\}} \gamma_{\mu}\left(\frac{K}{K^{2}-m_{f}^{2}}\right. \\
& \left.-\frac{\gamma_{5}[(K . n) \not h-(K . u) \not h]}{\left(K^{2}-m_{f}^{2}\right)^{2}} q_{f} B\right) \gamma^{\mu} \frac{1}{(P-K)^{2}} \\
\simeq & \Sigma^{B=0}(P, T)+\Sigma^{B \neq 0}(P, T) \equiv \Sigma^{0}+\Sigma^{B} .
\end{aligned}
$$

where $g$ is the QCD coupling constant, $C_{F}=4 / 3$ is the Casimir invariant of $S U(3)$ group, $T$ is the temperature of the system. The first term is the thermal bath contribution in the absence of a magnetic field $(B=0)$ whereas the second one is from the magnetized thermal bath.

Using (A4) in (21a) and (21b), the structure functions $a$ and $b$, respectively, become

$$
\begin{aligned}
& a\left(p_{0},|\vec{p}|\right)=\frac{1}{4} \frac{\operatorname{Tr}\left(\Sigma^{0} \not P\right)-(P . u) \operatorname{Tr}\left(\Sigma^{0} \not \iota\right)}{(P . u)^{2}-P^{2}}, \\
& b\left(p_{0},|\vec{p}|\right)=\frac{1}{4} \frac{-(P . u) \operatorname{Tr}\left(\Sigma^{0} \not P\right)+P^{2} \operatorname{Tr}\left(\Sigma^{0} \not \iota\right)}{(P . u)^{2}-P^{2}},
\end{aligned}
$$

where the contributions coming from $\Sigma^{B}$ vanish due to the trace of odd number of $\gamma$ matrices. Following the wellknown results in Ref. [69], one can write

$$
\begin{aligned}
& a\left(p_{0},|\vec{p}|\right)=-\frac{m_{\text {th }}^{2}}{|\vec{p}|^{2}} Q_{1}\left(\frac{p_{0}}{|\vec{p}|}\right), \\
& b\left(p_{0},|\vec{p}|\right)=\frac{m_{\text {th }}^{2}}{|\vec{p}|}\left[\frac{p_{0}}{|\vec{p}|} Q_{1}\left(\frac{p_{0}}{|\vec{p}|}\right)-Q_{0}\left(\frac{p_{0}}{|\vec{p}|}\right)\right],
\end{aligned}
$$

where the Legendre functions of the second kind read as

$$
\begin{aligned}
& Q_{0}(x)=\frac{1}{2} \ln \left(\frac{x+1}{x-1}\right), \\
& Q_{1}(x)=x Q_{0}(x)-1=\frac{x}{2} \ln \left(\frac{x+1}{x-1}\right)-1,
\end{aligned}
$$

and the thermal mass $[69,72]$ of the quark is given as

$$
m_{t h}^{2}=C_{F} \frac{g^{2} T^{2}}{8} .
$$

The thermal part of the self-energy in (A4) becomes

$$
\begin{aligned}
\Sigma^{B=0}(P, T) & \equiv \Sigma^{0}(P, T) \\
& =g^{2} C_{F} T \bigvee_{K} \gamma_{\mu} \frac{K}{K^{2}-m^{2}} \gamma^{\mu} \frac{1}{(P-K)^{2}} \\
& =-a\left(p_{0},|\vec{p}|\right) P-b\left(p_{0},|\vec{p}|\right) \not l .
\end{aligned}
$$

Again using (A4) in (21c) and (21d), the structure functions $b^{\prime}$ and $c^{\prime}$, respectively, become

$$
\begin{aligned}
& b^{\prime}=-\frac{1}{4} \operatorname{Tr}\left(\not h \gamma_{5} \Sigma^{B}\right), \\
& c^{\prime}=\frac{1}{4} \operatorname{Tr}\left(\not h \gamma_{5} \Sigma^{B}\right),
\end{aligned}
$$

where the contributions coming from $\Sigma^{0}$ vanish due to the trace of odd number of $\gamma$ matrices. For computing the above thermomagnetic structure functions, one needs to use the following two traces:

$$
\begin{aligned}
& \operatorname{Tr}\left[\not h \gamma_{5} \gamma_{\mu} \gamma_{5}[(K . n) \not h-(K . u) \not h] \gamma^{\mu}\right]=8(K . n), \\
& \operatorname{Tr}\left[\not h \gamma_{5} \gamma_{\mu} \gamma_{5}[(K . n) \not h-(K . u) \not h] \gamma^{\mu}\right]=8(K . u) .
\end{aligned}
$$

With this one can obtain

$$
\begin{aligned}
& b^{\prime}=2 g^{2} C_{F} T q_{f} B \bigcup_{\{K\}}(K . n) \Delta_{F}^{2}(K) \Delta_{B}(P-K), \\
& c^{\prime}=-2 g^{2} C_{F} T q_{f} B \bigcup_{\{K\}}(K . u) \Delta_{F}^{2}(K) \Delta_{B}(P-K),
\end{aligned}
$$

where the boson propagator in Saclay representation is given by

$$
\Delta_{B}(K)=-\int_{0}^{\beta} d \tau e^{k_{0} \tau} \tilde{\Delta}_{B}(\tau, k)
$$

and

$$
\begin{aligned}
\tilde{\Delta}_{B}(\tau, k) & =\sum_{k_{0}} e^{-k_{0} \tau} \Delta_{B}(K) \\
& =\frac{1}{2 \omega_{k}}\left\{\left[1+n_{B}\left(\omega_{k}\right)\right] e^{-\omega_{k} \tau}+n_{B}\left(\omega_{k}\right) e^{\omega_{k} \tau}\right\}
\end{aligned}
$$

where the sum is over $k_{0}=2 \pi i n T$ and $\omega_{k}^{2}=k^{2}+m_{f}^{2}$. Also the fermion propagator in Saclay representation reads

$$
\Delta_{F}(K)=-\int_{0}^{\beta} d \tau e^{k_{0} \tau} \tilde{\Delta}_{F}(\tau, k)
$$

and 


$$
\begin{aligned}
\tilde{\Delta}_{F}(\tau, k) & =\sum_{k_{0}} e^{-k_{0} \tau} \Delta_{F}(K) \\
& =\frac{1}{2 \omega_{k}}\left\{\left[1-n_{F}\left(\omega_{k}\right)\right] e^{-\omega_{k} \tau}-n_{F}\left(\omega_{k}\right) e^{\omega_{k} \tau}\right\}
\end{aligned}
$$

where the sum above is over $k_{0}=(2 n+1) \pi i T$. Now following HTL approximation in the presence of a magnetic field $[66,68]$ the (A14) and (A15) are simplified as

$$
\begin{aligned}
b^{\prime} & =-4 g^{2} C_{F} M^{2}\left(T, m_{f}, q_{f} B\right) \int \frac{d \Omega}{4 \pi} \frac{\hat{K} \cdot n}{P \cdot \hat{K}}, \\
c^{\prime} & =4 g^{2} C_{F} M^{2}\left(T, m_{f}, q_{f} B\right) \int \frac{d \Omega}{4 \pi} \frac{\hat{K} \cdot u}{P \cdot \hat{K}} .
\end{aligned}
$$

Using the results of the HTL angular integrations [67]

$$
\begin{gathered}
\int \frac{d \Omega}{4 \pi} \frac{\hat{K} \cdot u}{P \cdot \hat{K}}=\frac{1}{|\vec{p}|} Q_{0}\left(\frac{p^{0}}{|\vec{p}|}\right), \\
\int \frac{d \Omega}{4 \pi} \frac{\hat{K} \cdot n}{P \cdot \hat{K}}=-\frac{p^{3}}{|\vec{p}|^{2}} Q_{1}\left(\frac{p^{0}}{|\vec{p}|}\right),
\end{gathered}
$$

the thermomagnetic structures functions become

$$
\begin{aligned}
& b^{\prime}=4 g^{2} C_{F} M^{2}\left(T, m_{f}, q_{f} B\right) \frac{p^{3}}{|\vec{p}|^{2}} Q_{1}\left(\frac{p^{0}}{|\vec{p}|}\right), \\
& c^{\prime}=4 g^{2} C_{F} M^{2}\left(T, m_{f}, q_{f} B\right) \frac{1}{|\vec{p}|} Q_{0}\left(\frac{p^{0}}{|\vec{p}|}\right),
\end{aligned}
$$

with the magnetic mass is obtained as

$$
M^{2}\left(T, m_{f}, q_{f} B\right)=\frac{q_{f} B}{16 \pi^{2}}\left[\ln (2)-\frac{T}{m_{f}} \frac{\pi}{2}\right] .
$$

We note here that for $m_{f} \rightarrow 0$, the magnetic mass diverges but it can be regulated by the thermal mass $m_{t h}$ in (A8) as is done in Refs. [66,67]. Then the domain of applicability becomes $m_{t h}^{2}\left(\sim g^{2} T^{2}\right)<q_{f} B<T^{2}$ instead of $m_{f}^{2}<q_{f} B<T^{2}$.

The thermomagnetic part of the self-energy in (A4) becomes

$$
\begin{aligned}
\Sigma^{B \neq 0}(P, T) \equiv & \Sigma^{B}(P, T)=-g^{2} C_{F} T q_{f} B \bigvee \gamma_{\mu} \\
& \times \frac{\gamma_{5}[(K . n) \not h-(K . u) \not h]}{\left(K^{2}-m_{f}^{2}\right)^{2}} \gamma^{\mu} \frac{1}{(P-K)^{2}} \\
= & -b^{\prime}\left(p_{0},|\vec{p}|\right) \gamma_{5} \not h-c^{\prime}\left(p_{0},|\vec{p}|\right) \gamma_{5} \not h .
\end{aligned}
$$

Now combining (A9), (A21) and (A4), the general structure of quark self-energy in hot magnetized QCD becomes

$$
\begin{aligned}
\Sigma\left(p_{0},|\vec{p}|\right)= & -a\left(p_{0},|\vec{p}|\right) P\left(-b\left(p_{0},|\vec{p}|\right) \not h\right. \\
& -\gamma_{5} b^{\prime}\left(p_{0},|\vec{p}|\right) \not h-\gamma_{5} c^{\prime}\left(p_{0},|\vec{p}|\right) \not h .
\end{aligned}
$$

which agrees quite well with the general structure as discussed in (16) and also with results directly calculated in Refs. [66-68].

\section{APPENDIX B: SOLUTION OF THE MODIFIED DIRAC EQUATION AT LOWEST LANDAU LEVEL (LLL)}

At LLL, $l \rightarrow 0 \Rightarrow p_{\perp}=0$ and the effective Dirac equation becomes

$$
\begin{aligned}
& \left(\mathcal{P}_{+} \not \mathcal{L}+\mathcal{P}_{-} \not R\right) U=0 \\
& \left(\begin{array}{cc}
0 & R_{0}-\sigma^{3} R_{z} \\
L_{0}+\sigma^{3} L_{z} & 0
\end{array}\right) U=0,
\end{aligned}
$$

where $U=\left(\begin{array}{l}\psi_{L} \\ \psi_{R}\end{array}\right)$ with $\psi_{L(R)}$ are $2 \times 1$ blocks. Now, the condition for the nontrivial solution to exist is given as

$$
\begin{array}{cc}
\operatorname{det}\left(\begin{array}{cc}
0 & R_{0}-\sigma^{3} R_{z} \\
L_{0}+\sigma^{3} L_{z} & 0
\end{array}\right)=0 \\
{\left[\left(R_{0}\right)^{2}-\left(R_{z}\right)^{2}\right]\left[\left(L_{0}\right)^{2}-\left(L_{z}\right)^{2}\right]=0} \\
\text { or, } \quad R_{0}= \pm R_{z}, \quad L_{0}= \pm L_{z},
\end{array}
$$

(i) Case-I: For $R_{0}=R_{z}$, one can write (B1) as

$$
\begin{aligned}
& \left(\begin{array}{cccc}
0 & 0 & 0 & 0 \\
0 & 0 & 0 & 2 R_{z} \\
L_{0}+L_{z} & 0 & 0 & 0 \\
0 & L_{0}-L_{z} & 0 & 0
\end{array}\right) \\
& \cdot\left(\begin{array}{c}
\psi_{L}^{(1)} \\
\psi_{L}^{(2)} \\
\psi_{R}^{(1)} \\
\psi_{R}^{(2)}
\end{array}\right)=0,
\end{aligned}
$$

which leads to the following conditions:

$$
\begin{aligned}
2 R_{z} \psi_{R}^{(2)} & =0, \\
\left(L_{0}+L_{z}\right) \psi_{L}^{(1)} & =0, \\
\left(L_{0}-L_{z}\right) \psi_{L}^{(2)} & =0, \\
\psi_{R}^{(1)} & =\text { arbitrary } .
\end{aligned}
$$

For normalization, we choose only the nonzero component, $\psi_{R}^{(1)}=1$, which leads to 


$$
U_{R}^{(+)}=\left(\begin{array}{l}
0 \\
0 \\
1 \\
0
\end{array}\right) \text {. }
$$

Now, for $R_{0}=-R_{z}$, similarly, one can obtain

$$
U_{R}^{(-)}=\left(\begin{array}{l}
0 \\
0 \\
0 \\
1
\end{array}\right) .
$$

(ii) Case-II: For $L_{0}=L_{z}$, one gets

$$
U_{L}^{(+)}=\left(\begin{array}{l}
0 \\
1 \\
0 \\
0
\end{array}\right),
$$

whereas for $L_{0}=-L_{z}$, one finds

$$
U_{L}^{(-)}=\left(\begin{array}{l}
1 \\
0 \\
0 \\
0
\end{array}\right) .
$$

\section{APPENDIX C: VERIFICATION OF THE THREE-POINT FUNCTION FROM DIRECT CALCULATION}

In this appendix, we would verify the general structure of the temporal three-point function as obtained in sec. IV using the general structure of the self-energy.

We begin with the one-loop level three-point function in a hot magnetized medium in [67] within HTL approximation $[48,75]$ as

$\Gamma^{\mu}(P, K ; Q)=\gamma^{\mu}+\delta \Gamma_{\mathrm{HTL}}^{\mu}(P, K)+\delta \Gamma_{\mathrm{TM}}^{\mu}(P, K)$,

where the external four-momentum $Q=P-K$. The HTL correction part $[49,74,75]$ is given as

$$
\begin{aligned}
\delta \Gamma_{\mathrm{HTL}}^{\mu}(P, K) & =m_{t h}^{2} G^{\mu \nu} \gamma_{\nu} \\
& =m_{t h}^{2} \int \frac{d \Omega}{4 \pi} \frac{\hat{Y}^{\mu} \hat{Y}^{\nu}}{(P \cdot \hat{Y})(K \cdot \hat{Y})} \gamma_{\nu} \\
& =\delta \Gamma_{\mathrm{HTL}}^{\mu}(-P,-K),
\end{aligned}
$$

where $\hat{Y}_{\mu}=(1, \hat{y})$ is a lightlike four-vector, and the thermomagnetic (TM) correction part $[66,67]$ is given

$$
\begin{aligned}
\delta \Gamma_{\mathrm{TM}}^{\mu}(P, K)= & 4 \gamma_{5} g^{2} C_{F} M^{2} \int \frac{d \Omega}{4 \pi} \frac{1}{(P \cdot \hat{Y})(K \cdot \hat{Y})} \\
& \times[(\hat{Y} \cdot n) \not h-(\hat{Y} \cdot u) \not h] \hat{Y}^{\mu} .
\end{aligned}
$$

Now, choosing the temporal component of the thermomagnetic correction part of the three-point function and external three momentum $\vec{q}=0$, we get

$$
\begin{aligned}
& \left.\delta \Gamma_{\mathrm{TM}}^{0}(P, K)\right|_{\vec{q}=0} \\
& \quad=\gamma_{5} M^{\prime 2} \int \frac{d \Omega}{4 \pi} \frac{1}{(P \cdot \hat{Y})(K \cdot \hat{Y})}[(\hat{Y} \cdot n) \not h-(\hat{Y} \cdot u) \not h] \\
& \quad=\gamma_{5} M^{\prime 2} \int \frac{d \Omega}{4 \pi} \frac{1}{(P \cdot \hat{Y})(K \cdot \hat{Y})}\left[(\hat{Y} \cdot n) \gamma_{0}+(\hat{Y} \cdot u) \gamma^{3}\right]
\end{aligned}
$$

Along with this following identity:

$$
\left(\frac{1}{K \cdot \hat{Y}}-\frac{1}{P \cdot \hat{Y}}\right)=\frac{Q \cdot \hat{Y}}{(P \cdot \hat{Y})(K \cdot \hat{Y})}=\frac{q_{0}}{(P \cdot \hat{Y})(K \cdot \hat{Y})},
$$

and, (A16) and (A17), we one finally obtain

$$
\begin{aligned}
\left.\delta \Gamma_{\mathrm{TM}}^{0}(P, K)\right|_{\vec{q} \rightarrow} & =\frac{M^{\prime 2} p_{z}}{p^{2} q_{0}} \delta Q_{1} \gamma_{5} \gamma^{0}-\frac{M^{\prime 2}}{p q_{0}} \delta Q_{0} \gamma_{5} \gamma^{3} \\
& =-\frac{M^{\prime 2}}{p q_{0}}\left[\delta Q_{0} \gamma_{5}+\frac{p_{z}}{p} \delta Q_{1}\left(i \gamma^{1} \gamma^{2}\right)\right] \gamma^{3},
\end{aligned}
$$

where $\delta Q_{n}=Q_{n}\left(\frac{p_{0}}{p}\right)-Q_{n}\left(\frac{k_{0}}{p}\right)$. We note that this expression matches exactly with the expression obtained in (72) from the general structure of fermion self-energy.

\section{APPENDIX D: ANALYTICAL SOLUTION OF THE DISPERSION RELATIONS AND THE EFFECTIVE MASS IN LLL}

The dispersion relations at LLL can be written the Eqs. (31a) and (31b) as

$$
L_{L L L}^{2}=\left(\mathcal{A} p_{0}+\mathcal{B}_{+}\right)^{2}-\left(\mathcal{A} p_{z}+c^{\prime}\right)^{2}=L_{0}^{2}-L_{z}^{2}=0,
$$

$R_{L L L}^{2}=\left(\mathcal{A} p_{0}+\mathcal{B}_{-}\right)^{2}-\left(\mathcal{A} p_{z}-c^{\prime}\right)^{2}=R_{0}^{2}-R_{z}^{2}=0$,

each of which leads to two modes, respectively, as

$$
\begin{aligned}
L_{0} & = \pm L_{z} \\
\mathcal{A} p_{0}+\mathcal{B}_{+} & = \pm\left(\mathcal{A} p_{z}+c^{\prime}\right)
\end{aligned}
$$


and

$$
\begin{aligned}
R_{0} & = \pm R_{z} \\
\mathcal{A} p_{0}+\mathcal{B}_{-} & = \pm\left(\mathcal{A} p_{z}-c^{\prime}\right) .
\end{aligned}
$$

Below we try to get approximate analytical solution of these equations at small and high $p_{z}$ limits.

\section{Low $p_{z}$ limit}

In the low $p_{z}$ region, one needs to expand $a\left(p_{0},\left|p_{z}\right|\right)$, $b\left(p_{0},\left|p_{z}\right|\right), b^{\prime}\left(p_{0}, 0, p_{z}\right)$ and $c^{\prime}\left(p_{0},\left|p_{z}\right|\right)$ defined in (23a), (23b), (23c) and (23d), respectively, which depend on Legendre function of second kind $Q_{0}(x)$ and $Q_{1}(x)$ as given in Eqs. (A7a) and (A7b), respectively. The Legendre function $Q_{0}$ and structure coefficients are expanded in powers of $\frac{\left|p_{z}\right|}{p_{0}}$ as

$$
\begin{gathered}
Q_{0}\left(\frac{p_{0}}{\left|p_{z}\right|}\right)=\frac{\left|p_{z}\right|}{p_{0}}+\frac{1}{3} \frac{\left|p_{z}\right|^{3}}{p_{0}^{3}}+\frac{1}{5} \frac{\left|p_{z}\right|^{5}}{p_{0}^{5}}+\cdots \quad \text { (D4) } \\
a\left(p_{0},\left|p_{z}\right|\right)=-\frac{m_{t h}^{2}}{p_{0}^{2}}\left(\frac{1}{3}+\frac{1}{5} \frac{\left|p_{z}\right|^{2}}{p_{0}^{2}}+\cdots\right), \quad \text { (D5) } \\
b\left(p_{0},\left|p_{z}\right|\right)=-2 \frac{m_{t h}^{2}}{p_{0}}\left(\frac{1}{3}+\frac{1}{15} \frac{\left|p_{z}\right|^{2}}{p_{0}^{2}}+\cdots\right), \quad \text { (D6) } \\
b^{\prime}\left(p_{0}, 0, p_{z}\right)=4 g^{2} C_{F} M^{2}(T, m, q B) p_{z}\left(\frac{1}{3 p_{0}^{2}}+\frac{\left|p_{z}\right|^{2}}{5 p_{0}^{4}}+\cdots\right), \\
c^{\prime}\left(p_{0},\left|p_{z}\right|\right)=4 g^{2} C_{F} M^{2}(T, m, q B)\left(\frac{1}{p_{0}}+\frac{\left|p_{z}\right|^{2}}{p_{0}^{3}}+\cdots\right) .
\end{gathered}
$$

Now retaining the terms that are up to the order of $p_{z}$ in (D5), (D6), (D7), (D8), we obtain the following expressions for the dispersion relation of various modes:

(1) $L_{0}=L_{z}$ leads to a mode $L^{(+)}$as

$$
\omega_{L^{(+)}}\left(p_{z}\right)=m_{L L L}^{*+}+\frac{1}{3} p_{z}
$$

(2) $L_{0}=-L_{z}$ leads to a mode $L^{(-)}$as

$$
\omega_{L^{(-)}}\left(p_{z}\right)=m_{L L L}^{*-}-\frac{1}{3} p_{z} .
$$

(3) $R_{0}=R_{z}$ leads to a mode $R^{(+)}$as

$$
\omega_{R^{(+)}}\left(p_{z}\right)=m_{L L L}^{*-}+\frac{1}{3} p_{z}
$$

(4) $R_{0}=-R_{z}$ leads to a mode $R^{(-)}$as

$$
\omega_{R^{(-)}}\left(p_{z}\right)=m_{L L L}^{*+}-\frac{1}{3} p_{z}
$$

where the effective masses of various modes are given as $m_{L L L}^{* \pm}= \begin{cases}\sqrt{m_{t h}^{2}+4 g^{2} C_{F} M^{2}\left(T, M, q_{f} B\right)}, & \text { for } L^{(+)} \& R^{(-)}, \\ \sqrt{m_{t h}^{2}-4 g^{2} C_{F} M^{2}\left(T, M, q_{f} B\right)}, & \text { for } R^{(+)} \& L^{(-)} .\end{cases}$

\section{High $p_{z}$ limit}

We note that $p_{z}$ can be written as

$$
p_{z}= \begin{cases}\left|p_{z}\right|, & \text { for } p_{z}>0 \\ -\left|p_{z}\right| . & \text { for } p_{z}<0\end{cases}
$$

In high $p_{z}$ limit, we obtain

(i)

$$
\begin{aligned}
& {\left[1+a\left(p_{0},\left|p_{z}\right|\right)\right]\left(p_{0}-p_{z}\right)+b\left(p_{0},\left|p_{z}\right|\right)} \\
& \quad= \begin{cases}p_{0}-\left|p_{z}\right|-\frac{m_{t h}^{2}}{\left|p_{z}\right|}, & \text { for } p_{z}>0 \\
2\left|p_{z}\right|+\frac{m_{t h}^{2}}{\left|p_{z}\right|}-\frac{m_{t h}^{2}}{\left|p_{z}\right|} \ln \left(\frac{2\left|p_{z}\right|}{p_{0}-\left|p_{z}\right|}\right), & \text { for } p_{z}<0\end{cases}
\end{aligned}
$$

(ii)

$$
\begin{aligned}
& {\left[1+a\left(p_{0},\left|p_{z}\right|\right)\right]\left(p_{0}+p_{z}\right)+b\left(p_{0},\left|p_{z}\right|\right)} \\
& \quad= \begin{cases}2\left|p_{z}\right|+\frac{m_{t h}^{2}}{\left|p_{z}\right|}-\frac{m_{t h}^{2}}{\left|t_{z}\right|} \ln \left(\frac{2\left|p_{z}\right|}{p_{0}-\left|p_{z}\right|}\right), & \text { for } p_{z}>0 \\
p_{0}-\left|p_{z}\right|-\frac{m_{t h}^{2}}{\left|p_{z}\right|}, & \text { for } p_{z}<0\end{cases}
\end{aligned}
$$

(iii)

$$
\begin{aligned}
b^{\prime} & \left(p_{0}, 0, p_{z}\right)+c^{\prime}\left(p_{0},\left|p_{z}\right|\right) \\
\quad= & \begin{cases}\frac{4 g^{2} C_{F} M^{2}}{\left|p_{z}\right|} \ln \left(\frac{2\left|p_{z}\right|}{p_{0}-\left|p_{z}\right|}\right)-\frac{4 g^{2} C_{F} M^{2}}{\left|p_{z}\right|}, & \text { for } p_{z}>0 \\
\frac{4 g^{2} C_{F} M^{2}}{\left|p_{z}\right|} & \text { for } p_{z}<0\end{cases}
\end{aligned}
$$

(D16) 
(iv)

$$
\begin{aligned}
& b^{\prime}\left(p_{0}, 0, p_{z}\right)-c^{\prime}\left(p_{0},\left|p_{z}\right|\right) \\
& \quad= \begin{cases}-\frac{4 g^{2} C_{F} M^{2}}{\left|p_{z}\right|} & \text { for } p_{z}>0 \\
-\frac{4 g^{2} C_{F} M^{2}}{\left|p_{z}\right|} \ln \left(\frac{2\left|p_{z}\right|}{p_{0}-\left|p_{z}\right|}\right)+\frac{4 g^{2} C_{F} M^{2}}{\left|p_{z}\right|}, & \text { for } p_{z}<0\end{cases}
\end{aligned}
$$

(1) $L_{0}=L_{z}$ leads to a mode $L^{(+)}$:

For $p_{z}>0$,

$$
\omega_{L^{(+)}}\left(p_{z}\right)=\left|p_{z}\right|+\frac{\left(m_{L L L}^{*+}\right)^{2}}{\left|p_{z}\right|} .
$$

For $p_{z}<0$,

$\omega_{L^{(+)}}\left(p_{z}\right)=\left|p_{z}\right|+\frac{2\left|p_{z}\right|}{e} \exp \left(-\frac{2 p_{z}^{2}}{\left(m_{L L L}^{*+}\right)^{2}}\right)$.

(2) $L_{0}=-L_{z}$ leads to a mode $L^{(-)}$:

For $p_{z}>0$,

$\omega_{L^{(-)}}\left(p_{z}\right)=\left|p_{z}\right|+\frac{2\left|p_{z}\right|}{e} \exp \left(-\frac{2 p_{z}^{2}}{\left(m_{L L L}^{*-}\right)^{2}}\right)$.

For $p_{z}<0$,

$$
\omega_{L^{(-)}}\left(p_{z}\right)=\left|p_{z}\right|+\frac{\left(m_{L L L}^{*-}\right)^{2}}{\left|p_{z}\right|} .
$$

(3) $R_{0}=R_{z}$ leads to a mode $R^{(+)}$:

For $p_{z}>0$,

$$
\omega_{R^{(+)}}\left(p_{z}\right)=\left|p_{z}\right|+\frac{\left(m_{L L L}^{*-}\right)^{2}}{\left|p_{z}\right|} .
$$

For $p_{z}<0$,

$\omega_{R^{(+)}}\left(p_{z}\right)=\left|p_{z}\right|+\frac{2\left|p_{z}\right|}{e} \exp \left(-\frac{2 p_{z}^{2}}{\left(m_{L L L}^{*-}\right)^{2}}\right)$.

(4) $R_{0}=-R_{z}$ leads to a mode $R^{(-)}$:

For $p_{z}>0$,

$\omega_{R^{(-)}}\left(p_{z}\right)=\left|p_{z}\right|+\frac{2\left|p_{z}\right|}{e} \exp \left(-\frac{2 p_{z}^{2}}{\left(m_{L L L}^{*+}\right)^{2}}\right)$.

For $p_{z}<0$,

$$
\omega_{R^{(-)}}\left(p_{z}\right)=\left|p_{z}\right|+\frac{\left(m_{L L L}^{*+}\right)^{2}}{\left|p_{z}\right|} .
$$

Note that, in the high-momentum limit, the above dispersion relations are given in terms of absolute values of $p_{z}$, i.e., $\left|p_{z}\right|$.

We further note that the above dispersion relations in the absence of the magnetic field reduce to HTL results, where left- and right-handed are degenerate.

\section{APPENDIX E: RECOVERING HTL SPECTRAL FUNCTION}

One can easily get back to the HTL thermal spectral function from (77) by turning off the magnetic field, i.e., $B=0 \Rightarrow b^{\prime}=c^{\prime}=0$, and one gets the following simplifications:

$\left.g_{L}^{1}\right|_{B=0}=\left.g_{R}^{1}\right|_{B=0}=g^{1} ;\left.\quad g_{L}^{2}\right|_{B=0}=\left.g_{R}^{2}\right|_{B=0}=g^{2} ;$

$\left.g_{L}^{3}\right|_{B=0}=\left.g_{R}^{3}\right|_{B=0}=0$,

$\left.L^{2}\right|_{B=0}=\left.R^{2}\right|_{B=0}=H^{2} ;\left.\quad \omega_{L^{( \pm)}}\right|_{B=0}=\left.\omega_{R^{( \pm)}}\right|_{B=0}=\omega_{ \pm}$,

$\left.\rho_{L}^{1}\right|_{B=0}=\left.\rho_{R}^{1}\right|_{B=0}=\rho^{1} ;\left.\quad \rho_{L}^{2}\right|_{B=0}=\left.\rho_{R}^{2}\right|_{B=0}=\rho^{2}$,

$\left.\rho^{3}\right|_{B=0}=0$.

These implies that the spectral function can be written as

$$
\left.\rho\right|_{B=0}=\gamma^{0} \rho^{1}-(\gamma \cdot \hat{p}) \rho^{2} .
$$

Now the HTL spectral function $[49,73]$ is given by

$$
\begin{aligned}
\rho_{\mathrm{HTL}} & =\frac{1}{2}\left(\gamma^{0}-\gamma \cdot \hat{p}\right) \rho_{+}+\frac{1}{2}\left(\gamma^{0}+\gamma \cdot \hat{p}\right) \rho_{-} \\
& =\frac{1}{2} \gamma^{0}\left(\rho_{+}+\rho_{-}\right)-\frac{1}{2}(\gamma \cdot \hat{p})\left(\rho_{+}-\rho_{-}\right),
\end{aligned}
$$

where $\rho_{ \pm}$are the HTL spectral function. Since the spectral has both pole and cut part, comparing (E4) and (E5), one gets for the pole parts

$$
\begin{aligned}
& \left.\rho^{1}\right|_{\text {pole }} ^{\mathrm{B}=0}=\frac{1}{2}\left[\left.\rho_{+}\right|_{\text {pole }} ^{\mathrm{HTL}}+\left.\rho_{-}\right|_{\text {pole }} ^{\mathrm{HTL}}\right] \\
& \left.\rho^{2}\right|_{\text {pole }} ^{\mathrm{B}=0}=\frac{1}{2}\left[\left.\rho_{+}\right|_{\text {pole }} ^{\mathrm{HTL}}-\left.\rho_{-}\right|_{\text {pole }} ^{\mathrm{HTL}}\right],
\end{aligned}
$$

and for the cut parts

$$
\begin{aligned}
& \left.\beta^{1}\right|_{\text {cut }} ^{\mathrm{B}=0}=\frac{1}{2}\left[\left.\beta_{+}\right|_{\mathrm{cut}} ^{\mathrm{HTL}}+\left.\beta_{-}\right|_{\mathrm{cut}} ^{\mathrm{HTL}}\right] \\
& \left.\beta^{2}\right|_{\mathrm{cut}} ^{\mathrm{B}=0}=\frac{1}{2}\left[\left.\beta_{+}\right|_{\mathrm{cut}} ^{\mathrm{HTL}}-\left.\beta_{-}\right|_{\mathrm{cut}} ^{\mathrm{HTL}}\right] .
\end{aligned}
$$

Now one can obtain either (78) or (79): 


$$
\begin{aligned}
\left.\rho^{1}\right|_{\text {pole }} ^{\mathrm{B}=0}= & {\left[Z_{+}^{i+} \delta\left(p_{0}-\omega_{+}\right)+Z_{+}^{i-} \delta\left(p_{0}+\omega_{+}\right)\right] } \\
& +\left[Z_{-}^{1+} \delta\left(p_{0}-\omega_{-}\right)+Z_{-}^{1-} \delta\left(p_{0}+\omega_{-}\right)\right] .
\end{aligned}
$$

When the magnetic field is turned off, the different residues can be read from their analytical expressions as given in Sec. V as

$$
\begin{aligned}
& Z_{+}^{1+}=Z_{+}^{1-}=\frac{\omega_{+}^{2}-p^{2}}{4 m_{t h}^{2}}=\frac{1}{2} Z_{+}, \\
& Z_{-}^{1+}=Z_{-}^{1+}=\frac{\omega_{-}^{2}-p^{2}}{4 m_{t h}^{2}}=\frac{1}{2} Z_{-},
\end{aligned}
$$

where $Z_{ \pm}=\left(\omega_{ \pm}^{2}-p^{2}\right) / 2 m_{t h}^{2}$ are the residues corresponding to the modes $\omega^{ \pm}$. Using these, one can write (E10) as

$$
\begin{aligned}
\left.\rho^{1}\right|_{\text {pole }} ^{\mathrm{B}=0}= & \frac{1}{2}\left[Z_{+} \delta\left(p_{0}-\omega_{+}\right)+Z_{+} \delta\left(p_{0}+\omega_{+}\right)\right] \\
& +\frac{1}{2}\left[Z_{-} \delta\left(p_{0}-\omega_{-}\right)+Z_{-} \delta\left(p_{0}+\omega_{-}\right)\right] \\
= & \frac{1}{2}\left[Z_{+} \delta\left(p_{0}-\omega_{+}\right)+Z_{-} \delta\left(p_{0}+\omega_{-}\right)\right] \\
& +\frac{1}{2}\left[Z_{-} \delta\left(p_{0}-\omega_{-}\right)+Z_{+} \delta\left(p_{0}+\omega_{+}\right)\right] \\
= & \frac{1}{2}\left[\left.\rho_{+}\right|_{\text {pole }} ^{\mathrm{HTL}}+\left.\rho_{-}\right|_{\text {pole }} ^{\mathrm{HTL}}\right],
\end{aligned}
$$

which agrees with (E6).

Now the other nonzero component of the spectral function can be reduced as

$$
\begin{aligned}
\left.\rho^{2}\right|_{\text {pole }} ^{\mathrm{B}=0}= & {\left[Z_{+}^{2+} \delta\left(p_{0}-\omega_{+}\right)+Z_{+}^{2-} \delta\left(p_{0}+\omega_{+}\right)\right] } \\
& +\left[Z_{-}^{2+} \delta\left(p_{0}-\omega_{-}\right)+Z_{-}^{2-} \delta\left(p_{0}+\omega_{-}\right)\right],
\end{aligned}
$$

along with the remaining nonzero residues as

$$
Z_{+}^{2+}=-Z_{+}^{2-}=\frac{\omega_{+}^{2}-p^{2}}{4 m_{t h}^{2}} \times \frac{\omega_{+} m_{t h}^{2} \log \left(\frac{\omega_{+}+p}{\omega_{+}-p}\right)-2 p\left(m_{t h}^{2}+p^{2}\right)}{2 p^{2} \omega_{+}-p m_{t h}^{2} \log \left(\frac{\omega_{+}+p}{\omega^{+}-p}\right)}=\frac{\omega_{+}^{2}-p^{2}}{4 m_{t h}^{2}}=\frac{1}{2} Z_{+},
$$

and

$$
Z_{-}^{2+}=-Z_{-}^{2-}=\frac{\omega_{-}^{2}-p^{2}}{4 m_{t h}^{2}} \times \frac{\omega_{-} m_{t h}^{2} \log \left(\frac{\omega_{-}+p}{\omega^{-}-p}\right)-2 p\left(m_{t h}^{2}+p^{2}\right)}{2 p^{2} \omega_{-}-p m_{t h}^{2} \log \left(\frac{\omega_{-}+p}{\omega_{-}-p}\right)}=\frac{\omega_{-}^{2}-p^{2}}{4 m_{t h}^{2}}=\frac{1}{2} Z_{-} .
$$

Note that we have used the respective dispersion relations coming from $H^{2}=0$, in the last line of (E15) and (E16) for further simplifications. Now (E14) can be rewritten as

$$
\begin{aligned}
\left.\rho^{2}\right|_{\text {pole }} ^{\mathrm{B}=0}= & \frac{1}{2}\left[Z_{+} \delta\left(p_{0}-\omega_{+}\right)-Z_{+} \delta\left(p_{0}+\omega_{+}\right)\right] \\
& -\frac{1}{2}\left[Z_{-} \delta\left(p_{0}-\omega_{-}\right)-Z_{-} \delta\left(p_{0}+\omega_{-}\right)\right] \\
= & \frac{1}{2}\left[Z_{+} \delta\left(p_{0}-\omega_{+}\right)+Z_{-} \delta\left(p_{0}+\omega_{-}\right)\right] \\
& -\frac{1}{2}\left[Z_{-} \delta\left(p_{0}-\omega_{-}\right)+Z_{+} \delta\left(p_{0}+\omega_{+}\right)\right] \\
= & \frac{1}{2}\left[\left.\rho_{+}\right|_{\text {pole }} ^{\mathrm{HTL}}-\left.\rho_{-}\right|_{\text {pole }} ^{\mathrm{HTL}}\right],
\end{aligned}
$$

$$
\beta^{2}=\frac{1}{\pi} \Theta\left(p^{2}-p_{0}^{2}\right) \frac{\operatorname{Im}\left(g^{2}\right) \operatorname{Re}\left(H^{2}\right)-\operatorname{Im}\left(H^{2}\right) \operatorname{Re}\left(g^{2}\right)}{\left|H^{2}\right|^{2}},
$$

where, for the zero magnetic field case,

$$
H^{2}=\left.L^{2}\right|_{B=0}=\left.R^{2}\right|_{B=0}=\left(g^{1}+g^{2}\right)\left(g^{1}-g^{2}\right)=H_{-} H_{+},
$$

where following the same convention as before $H_{-}=$ $g^{1}+g^{2}$ and $H_{+}=g^{1}-g^{2}$.

The real and imaginary parts of $H^{2}$ can be written in terms of $H_{-}$and $H_{+}$as
In the absence of a magnetic field, one can write the cut parts from the general expression of $\beta_{L}^{i}$ in (82) as 


$$
\begin{aligned}
\operatorname{Re}\left(H^{2}\right)+i \operatorname{Im}\left(H^{2}\right) & =\left(\operatorname{Re}\left(H_{-}\right)+i \operatorname{Im}\left(H_{-}\right)\right)\left(\operatorname{Re}\left(H_{+}\right)+i \operatorname{Im}\left(H_{+}\right)\right) \\
& =\left[\operatorname{Re}\left(H_{-}\right) \operatorname{Re}\left(H_{+}\right)-\operatorname{Im}\left(H_{-}\right) \operatorname{Im}\left(H_{+}\right)\right]+i\left[\operatorname{Re}\left(H_{-}\right) \operatorname{Im}\left(H_{+}\right)+\operatorname{Re}\left(H_{+}\right) \operatorname{Im}\left(H_{-}\right)\right] .
\end{aligned}
$$

Now we can write down

$$
\begin{aligned}
\beta^{1}+\beta^{2} & =\frac{1}{\pi} \Theta\left(p^{2}-p_{0}^{2}\right) \frac{\left(\operatorname{Im}\left(g^{1}\right)+\operatorname{Im}\left(g^{2}\right)\right) \operatorname{Re}\left(H^{2}\right)-\operatorname{Im}\left(H^{2}\right)\left(\operatorname{Re}\left(g^{1}\right)+\operatorname{Re}\left(g^{2}\right)\right)}{\left|H_{-}\right|^{2}\left|H_{+}\right|^{2}} \\
& =\frac{1}{\pi} \Theta\left(p^{2}-p_{0}^{2}\right) \frac{\operatorname{Im}\left(H_{-}\right) \operatorname{Re}\left(H^{2}\right)-\operatorname{Im}\left(H^{2}\right) \operatorname{Re}\left(H_{-}\right)}{\left|H_{1}\right|^{2}\left|H_{2}\right|^{2}} \\
& =-\frac{1}{\pi} \Theta\left(p^{2}-p_{0}^{2}\right) \frac{\operatorname{Im}\left(H_{+}\right)}{\left|H_{+}\right|^{2}} \\
& =-\frac{\frac{1}{2 p} m_{t h}^{2}\left(1-\frac{p_{0}}{p}\right)}{\left[\left\{p_{0}-p+\frac{m_{t h}^{2}}{2 p}\left(\left(1-\frac{p_{0}}{p}\right) \log \left|\frac{p_{0}+p}{p_{0}-p}\right|+2\right)\right\}^{2}+\frac{\pi^{2} m_{t h}^{4}}{4 p^{2}}\left(1-\frac{p_{0}}{p}\right)^{2}\right]} \\
& =\beta_{+} .
\end{aligned}
$$

and similarly

$$
\begin{aligned}
\beta^{1}-\beta^{2} & =-\frac{1}{\pi} \Theta\left(p^{2}-p_{0}^{2}\right) \frac{\operatorname{Im}\left(H_{-}\right)}{\left|H_{-}\right|^{2}}, \\
& =-\frac{\frac{1}{2 p} m_{t h}^{2}\left(1+\frac{p_{0}}{p}\right)}{\left[\left\{p_{0}+p-\frac{m_{t h}^{2}}{2 p}\left(\left(1+\frac{p_{0}}{p}\right) \log \left|\frac{p_{0}+p}{p_{0}-p}\right|-2\right)\right\}^{2}+\frac{\pi^{2} m_{\text {th }}^{4}}{4 p^{2}}\left(1+\frac{p_{0}}{p}\right)^{2}\right]}, \\
& =\beta_{-},
\end{aligned}
$$

where both (E22) and (E23) agree with the HTL cut parts [49,73].

[1] A. Adare et al. (PHENIX), Phys. Rev. Lett. 109, 122302 (2012).

[2] V. Skokov, A. Yu. Illarionov, and V. Toneev, Int. J. Mod. Phys. A 24, 5925 (2009).

[3] V. de la Incera, AIP Conf. Proc. 1361, 74 (2011) .

[4] D. Bandyopadhyay, S. Chakrabarty, and S. Pal, Phys. Rev. Lett. 79, 2176 (1997).

[5] S. Chakrabarty, D. Bandyopadhyay, and S. Pal, Phys. Rev. Lett. 78, 2898 (1997).

[6] J. O. Andersen, W. R. Naylor, and A. Tranberg, Rev. Mod. Phys. 88, 025001 (2016).

[7] G. Basar, D. Kharzeev, D. Kharzeev, and V. Skokov, Phys. Rev. Lett. 109, 202303 (2012).

[8] N. Sadooghi and F. Taghinavaz, Ann. Phys. (Amsterdam) 376, 218 (2017).

[9] A. Bandyopadhyay, C. A. Islam, and M. G. Mustafa, Phys. Rev. D 94, 114034 (2016).

[10] A. Bandyopadhyay and S. Mallik, Phys. Rev. D 95, 074019 (2017).

[11] K. Tuchin, Phys. Rev. C 88, 024911 (2013).

[12] K. Tuchin, Phys. Rev. C 87, 024912 (2013).

[13] K. Tuchin, Phys. Rev. C 88, 024910 (2013).
[14] D. E. Kharzeev, Prog. Part. Nucl. Phys. 75, 133 (2014).

[15] K. Fukushima, Lect. Notes Phys. 871, 241 (2013).

[16] G. Basar and G. V. Dunne, Lect. Notes Phys. 871, 261 (2013).

[17] V. P. Gusynin, V. A. Miransky, and I. A. Shovkovy, Nucl. Phys. B462, 249 (1996).

[18] I. A. Shovkovy, Lect. Notes Phys. 871, 13 (2013).

[19] F. Preis, A. Rebhan, and A. Schmitt, Lect. Notes Phys. 871, 51 (2013).

[20] R. L. S. Farias, K. P. Gomes, G. I. Krein, and M. B. Pinto, Phys. Rev. C 90, 025203 (2014).

[21] M. N. Chernodub, Lect. Notes Phys. 871, 143 (2013).

[22] E. S. Fraga, Lect. Notes Phys. 871, 121 (2013).

[23] D. E. Kharzeev, L. D. McLerran, and H. J. Warringa, Nucl. Phys. A803, 227 (2008).

[24] K. Landsteiner, E. Megias, and F. Pena-Benitez, Lect. Notes Phys. 871, 433 (2013).

[25] K. Hattori and K. Itakura, Ann. Phys. (Amsterdam) 330, 23 (2013).

[26] K. Hattori and K. Itakura, Ann. Phys. (Amsterdam) 334, 58 (2013). 
[27] S. Fayazbakhsh, S. Sadeghian, and N. Sadooghi, Phys. Rev. D 86, 085042 (2012).

[28] S. Fayazbakhsh and N. Sadooghi, Phys. Rev. D 88, 065030 (2013).

[29] M. Strickland, V. Dexheimer, and D. P. Menezes, Phys. Rev. D 86, 125032 (2012).

[30] J. O. Andersen, J. High Energy Phys. 10 (2012) 005.

[31] K. A. Mamo, J. High Energy Phys. 08 (2013) 083.

[32] N. Sadooghi and F. Taghinavaz, Phys. Rev. D 92, 025006 (2015).

[33] P. Elmfors, D. Persson, and B.-S. Skagerstam, Nucl. Phys. B464, 153 (1996).

[34] K. Bhattacharya and P. B. Pal, Pramana 62, 1041 (2004).

[35] K. Bhattacharya and P. B. Pal, Proc. Indian Natl. Acad. Sci. A U.S.A. 70, 145 (2004).

[36] A. K. Ganguly, S. Konar, and P. B. Pal, Phys. Rev. D 60, 105014 (1999).

[37] J. C. D’Olivo, J. F. Nieves, and S. Sahu, Phys. Rev. D 67, 025018 (2003).

[38] J. Schwinger, Phys. Rev. 82, 664 (1951).

[39] W.-y. Tsai, Phys. Rev. D 10, 1342 (1974).

[40] W.-y. Tsai, Phys. Rev. D 10, 2699 (1974).

[41] T.-K. Chyi, C.-W. Hwang, W. F. Kao, G.-L. Lin, K.-W. Ng, and J.-J. Tseng, Phys. Rev. D 62, 105014 (2000).

[42] S. P. Adhya, M. Mandal, S. Biswas, and P. K. Roy, Phys. Rev. D 93, 074033 (2016).

[43] A. Mukherjee, S. Ghosh, M. Mandal, P. Roy, and S. Sarkar, Phys. Rev. D 96, 016024 (2017).

[44] S. Ghosh, A. Mukherjee, M. Mandal, S. Sarkar, and P. Roy, Phys. Rev. D 94, 094043 (2016).

[45] A. Bandyopadhyay and S. Mallik, arXiv:1610.07887.

[46] S. Ghosh, A. Mukherjee, M. Mandal, S. Sarkar, and P. Roy, arXiv:1704.05319.

[47] J. O. Andersen, E. Braaten, and M. Strickland, Phys. Rev. Lett. 83, 2139 (1999).

[48] E. Braaten and R. D. Pisarski, Nucl. Phys. B337, 569 (1990).

[49] E. Braaten, R. D. Pisarski, and T. C. Yuan, Phys. Rev. Lett. 64, 2242 (1990).

[50] E. Braaten and R. D. Pisarski, Phys. Rev. D 42, 2156 (1990).

[51] N. Haque, M. G. Mustafa, and M. H. Thoma, Phys. Rev. D 84, 054009 (2011).
[52] N. Haque and M. G. Mustafa, Nucl. Phys. A862, 271 (2011).

[53] N. Haque and M. G. Mustafa, arXiv:1007.2076.

[54] J. O. Andersen, E. Braaten, E. Petitgirard, and M. Strickland, Phys. Rev. D 66, 085016 (2002).

[55] J. O. Andersen, E. Petitgirard, and M. Strickland, Phys. Rev. D 70, 045001 (2004).

[56] N. Haque, M. G. Mustafa, and M. Strickland, Phys. Rev. D 87, 105007 (2013).

[57] N. Haque, M. G. Mustafa, and M. Strickland, J. High Energy Phys. 07 (2013) 184.

[58] J. O. Andersen, M. Strickland, and N. Su, Phys. Rev. Lett. 104, 122003 (2010).

[59] J. O. Andersen, M. Strickland, and N. Su, J. High Energy Phys. 08 (2010) 113.

[60] J. O. Andersen, L. E. Leganger, M. Strickland, and N. Su, Phys. Lett. B 696, 468 (2011).

[61] J. O. Andersen, L. E. Leganger, M. Strickland, and N. Su, J. High Energy Phys. 08 (2011) 053.

[62] J. O. Andersen, L. E. Leganger, M. Strickland, and N. Su, Phys. Rev. D 84, 087703 (2011).

[63] N. Haque, J. O. Andersen, M. G. Mustafa, M. Strickland, and N. Su, Phys. Rev. D 89, 061701 (2014).

[64] N. Haque, A. Bandyopadhyay, J. O. Andersen, M. G. Mustafa, M. Strickland, and N. Su, J. High Energy Phys. 05 (2014) 027.

[65] M. G. Mustafa, M. H. Thoma, and P. Chakraborty, Phys. Rev. C 71, 017901 (2005).

[66] A. Ayala, J. J. Cobos-Martínez, M. Loewe, M. E. TejedaYeomans, and R. Zamora, Phys. Rev. D 91, 016007 (2015).

[67] N. Haque, Phys. Rev. D 96, 014019 (2017).

[68] A. Bandyopadhyay, N. Haque, and M. G. Mustafa, arXiv: 1702.02875 .

[69] H. A. Weldon, Phys. Rev. D 26, 2789 (1982).

[70] H. A. Weldon, Phys. Rev. D 26, 1394 (1982).

[71] H. A. Weldon, Phys. Rev. D 61, 036003 (2000).

[72] M. L. Bellac, Thermal Field Theory (Cambridge University Press, Cambridge, England, 2011).

[73] F. Karsch, M. G. Mustafa, and M. H. Thoma, Phys. Lett. B 497, 249 (2001).

[74] P. Chakraborty, M. G. Mustafa, and M. H. Thoma, Eur. Phys. J. C 23, 591 (2002).

[75] J. Frenkel and J. C. Taylor, Nucl. Phys. B334, 199 (1990). 